\title{
Delivery strategies to control inflammatory response: Modulating M1-M2 polarization in tissue engineering applications
}

Mario Moisés Alvarez ${ }^{1,2,3,4, \S}$, Julie C. Liu ${ }^{1,2,5, \S}$, Grissel Trujillo-de Santiago ${ }^{1,2,3,4}$, Byung-Hyun Cha $^{1,2,7}$, Ajaykumar Vishwakarma ${ }^{1,2}$, Amir Ghaemmaghami ${ }^{6}$, and Ali Khademhosseini ${ }^{1,2,3,7,8,9}$

(1) Biomaterials Innovation Research Center, Brigham and Women's Hospital, Harvard Medical School, Boston, Massachusetts, United States of America

(2) Harvard-Massachusetts Institute of Technology Division of Health Sciences and Technology, Massachusetts Institute of Technology, Cambridge, Massachusetts, United States of America

(3) Microsystems Technologies Laboratories, Massachusetts Institute of Technology, Cambridge, 02139, MA, USA

(4) Centro de Biotecnología-FEMSA, Tecnológico de Monterrey at Monterrey, Monterrey, Nuevo León, México

(5) School of Chemical Engineering and Weldon School of Biomedical Engineering, Purdue University, West Lafayette, Indiana, United States of America

(6) Division of Immunology, School of Life Sciences, Faculty of Medicine and Health Sciences, Queen's Medical Centre, University of Nottingham, Nottingham, United Kingdom

(7) Wyss Institute for Biologically Inspired Engineering, Harvard University, Boston, Massachusetts, United States of America

(8) Department of Bioindustrial Technologies, College of Animal Bioscience and Technology, Konkuk University, Hwayang-dong, Gwangjin-gu, Seoul 143-701, Republic of Korea

(9) Department of Physics, King Abdulaziz University, Jeddah, Saudi Arabia

${ }^{\S}$ These authors contributed equally to this work.

Ali Khademhosseini (corresponding author), e-mail: alik@ rics.bwh.harvard.edu

Abstract: Macrophages are key players in many physiological scenarios including tissue homeostasis. In response to injury, typically the balance between macrophage sub-populations shifts from an M1 phenotype (pro-inflammatory) to an M2 phenotype (anti-inflammatory). In 
tissue engineering scenarios, after implantation of any device, it is desirable to exercise control on this M1-M2 progression and to ensure a timely and smooth transition from the inflammatory to the healing stage. In this review, we briefly introduce the current state of knowledge regarding macrophage function and nomenclature. Next, we discuss the use of controlled release strategies to tune the balance between the M1 and M2 phenotypes in the context of tissue engineering applications. We discuss recent literature related to the release of anti-inflammatory molecules (including nucleic acids) and the sequential release of cytokines to promote a timely M1-M2 shift. In addition, we describe the use of macrophages as controlled release agents upon stimulation by physical and/or mechanical cues provided by scaffolds. Moreover, we discuss current and future applications of "smart" implantable scaffolds capable of controlling the cascade of biochemical events related to healing and vascularization. Finally, we provide our opinion on the current challenges and the future research directions to improve our understanding of the M1-M2 macrophage balance and properly exploit it in tissue engineering and regenerative medicine applications.

Key words: macrophages, M1 and M2, inflammation, controlled release, anti-inflammatory, tissue engineering

Submitted to

Journal of Controlled Release

\section{Introduction: From inert to bioactive to smart implants}

Developing strategies that control the immune response and enhance engraftment is a universal challenge in the clinical translation of tissue engineered products $[1,2]$. In the past, most approaches have focused on minimizing the inflammatory response to bioengineered constructs. However, recent studies demonstrate that fine-tuning the balance of pro- and antiinflammatory responses can benefit tissue engineering strategies by supporting endogenous healing and regeneration. Thus, understanding the role of the immune system and its importance in the context of regenerative medicine is now considered of great translational value and yet remains largely unexplored. 
The immune response to implantation of any foreign material (e.g., a tissue engineering scaffold, a biomedical device, a drug releasing implant, etc.) is complex and depends on multiple factors, including the nature of the implant (its material, surface topography, physical and chemical properties, size and shape, etc.) and the state of the host tissue [3-5]. Soon after implantation, a series of immunological and vascular reactions are triggered on the foreign material surface. Inflammatory mediators, cytokines, reactive oxygen species, and growth or angiogenic factors secreted by immune cells all influence a material's performance. Depending on the reactivity of the immune cells to the material, the spatial-temporal release of bioactive agents and the end-stage result may vary. One example is the foreign body response (FBR), in which macrophages and foreign body giant cells play a primary role [3]. In the FBR, tissue regeneration is limited and a fibrotic capsule surrounds the biomaterial, which results in the accumulation of toxic byproducts of phagocytosis at the implant site.

In the context of surgical and tissue engineering implants, biomedical devices, and drug releasing scaffolds, the initial aim of biomedical engineers was to identify and develop inert and biocompatible materials that could "evade" the immune response (or render the implant "invisible" to the immune system). Later, the development of bioactive and biodegradable materials (and implants) dominated this research field [5,6]. Currently, the focus has gone one step forward, and the aim now is to design and develop smart scaffolds and materials for tissue engineering applications-materials that can exert a positive action on the process of implant integration and healing [4]. A better understanding of the human immune response to tissue engineered products has aided the development of micro- and nanoengineered 3D environments that can tightly control the spatial and temporal release of chemical cues to mitigate inflammation and/or promote tissue regeneration. 
In this contribution, we discuss approaches in which controlled release strategies are directly used to attenuate inflammation or to favor a timely transition to an M2 phenotype. In addition, we describe scenarios in which macrophages are used as controlled release vehicles to deliver specific cytokines and other molecules in response to chemical or physical cues from a biomaterial.

\section{The evolving concept of M1-M2 polarization}

Macrophages are key actors during the host immune response in many different physiological contexts in both health and disease (Figure 1A) [7,8] and are involved in autoimmune and inflammatory diseases $[9,10]$, asthma and allergies[11,12], cancer [13-16], and resistance to infections $[17,18]$. Macrophages not only influence the innate and adaptive immune capacity but also play an important role in tissue homeostasis [7]. For example, macrophages generate a chemical environment that alerts the body in case of injury and also promotes healing at the cellular and tissue level [19].

In the context of tissue engineering and regenerative medicine, the implantation of a scaffold (or any foreign material) triggers a plethora of biochemical signals wherein macrophages act as protagonists in the processes of inflammation and healing (Figure 1B and 2A). In the first stage, inflammation occurs as a natural response to the presence of the exogenous material. Pro-inflammatory cytokines (e.g., tumor necrosis factor (TNF $\alpha$ ), interleukin (IL)-1, etc.) alert the immune system at a local and systemic level and recruit monocytes (among other cell types) to the vicinity of the implant. Circulating monocytes differentiate into macrophages in response to this set of pro-inflammatory chemical signals. Until recently, the prevailing opinion was that this differentiation from monocytes was the only origin for macrophages, but strong evidence now indicates that many tissue-resident macrophages originate at the embryonic stage, persist, and proliferate into adulthood (see Figure 1A) [20-24]. The 
relative contribution of the macrophages differentiated from monocytes versus tissue-resident macrophages is still unclear and remains an active topic of study $[21,23,24]$.
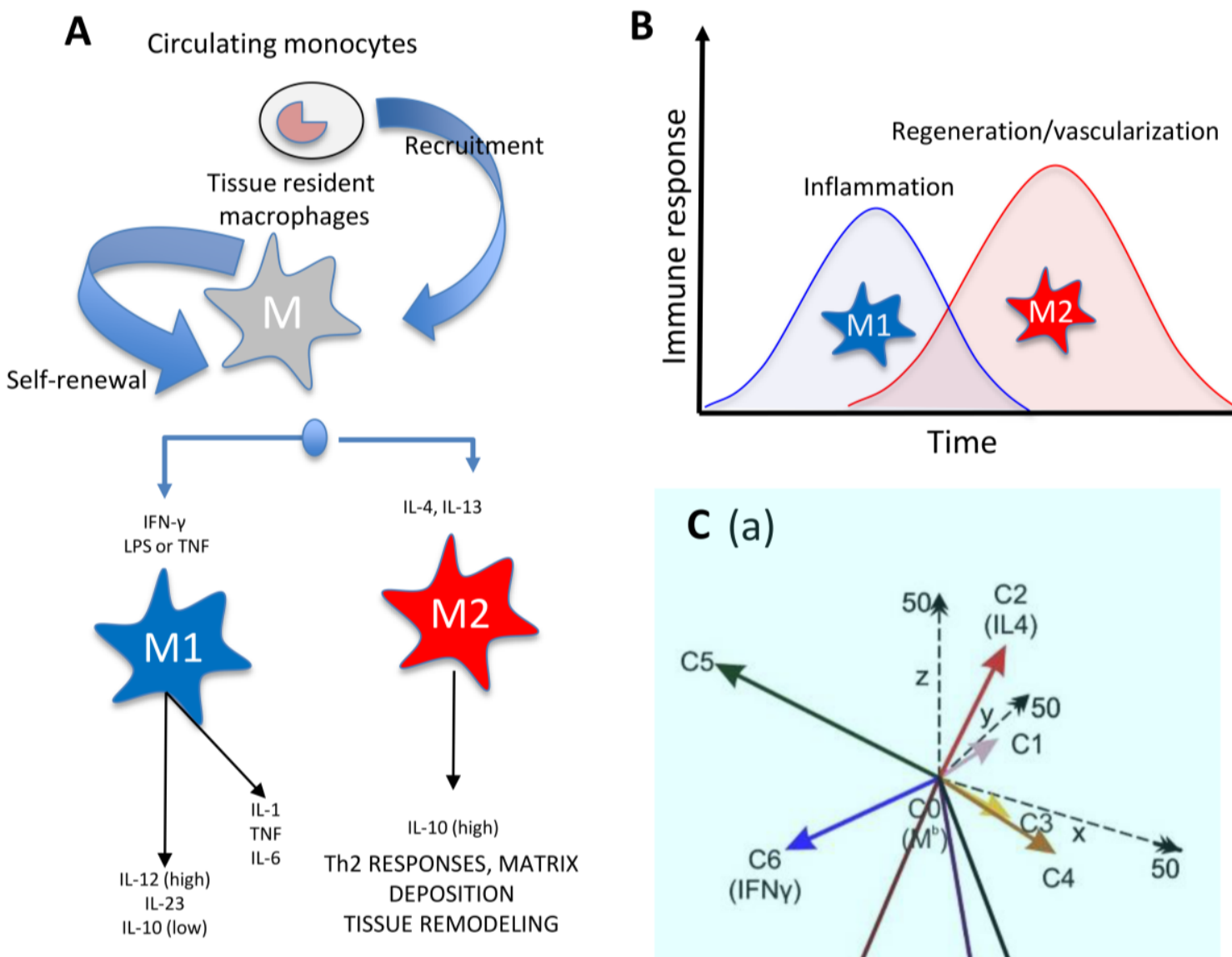

INFLAMMATION
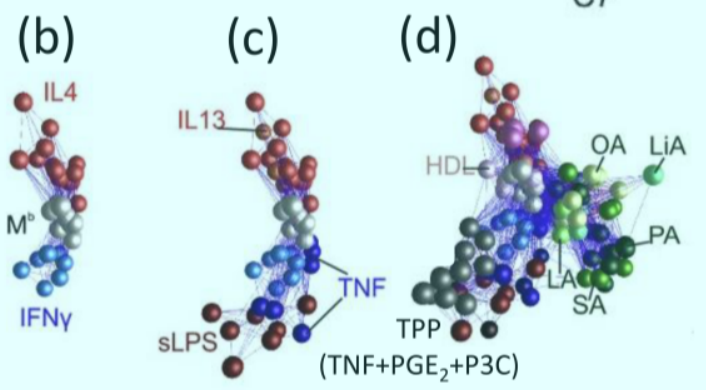

(d)

C (a)

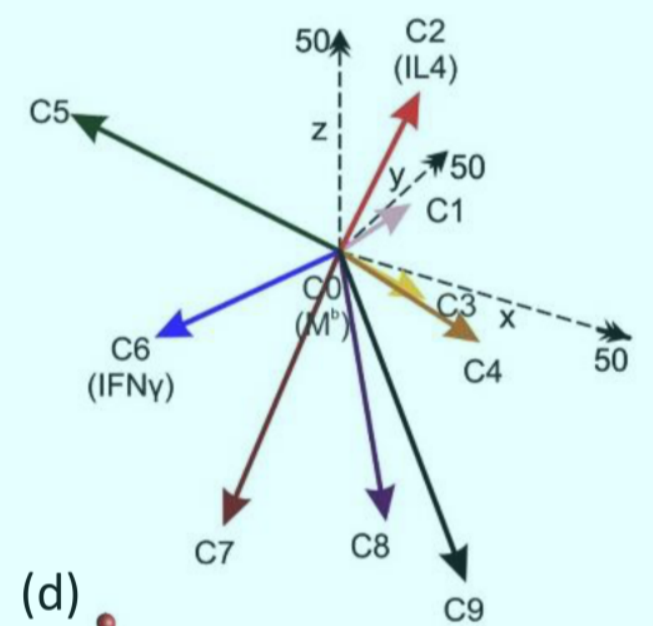

Figure 1: Prevalent nomenclature and timing of macrophages. (A) Most adult tissues contain naïve tissueresident macrophages that originated in the embryonic stage [126]. In addition, circulating monocytes can be recruited to a site of injury where they differentiate into various macrophage phenotypes. According to the most prevalent nomenclature, two macrophage phenotypes have been recognized. The M1 phenotype (classically activated or pro-inflammatory) is activated by IFN $\gamma$ and LPS. The M2 phenotype (alternatively activated or antiinflammatory) is activated by IL-4 and IL-13. Modified from Mantovani et al., 2004 [41]. (B) Parallel events occur 
during bioengineered tissue formation. The biomaterial implantation (with or without cells) will trigger an immune response, which leads to recruitment of various immune cells and macrophage infiltration. At this stage, a dominant M1 macrophage phenotype should be expected, and pro-inflammatory stimuli (required during the first stage of an injury) are upregulated. Next, a timely progression toward an anti-inflammatory chemical scenario could be beneficial for more rapid and smoother implant-tissue integration and healing; thus the M2 phenotype is more desirable in the second stage. (C) A recent report by Xue et al. reported many more than the two widely recognized (M1 and M2) phenotypes: (a) Nine different macrophage phenotypes were identified in human macrophages based on their transcriptome signatures upon activation by different chemical cues. When macrophages were (b) activated with known M1 or M2 chemical cues or (c) with M1 or M2 related chemical signals, they displayed a biochemical behavior consistent with the M1-M2 polarization model. (d) However, activation with other chemical cues (e.g., free fatty acids, high density lipoprotein (HDL), or molecules associated with chronic inflammation) resulted in seven other distinct macrophage phenotypes (a multi-axis spectrum; C1, C3, C4, C5, C7, C8, C9). C2 and C6 are consistent with the expression profile of M2 and M1 phenotypes, respectively. Adapted with permission from Xue et al., 2014 [27].

In general, macrophages remain at the tissue-implant interface for the entire lifespan of the implant and become key mediators of inflammation as well as immune and foreign body responses. One key function of macrophages is degrading materials through phagocytosis. Macrophages also play a critical role in tissue regeneration through regulating cells involved in the wound healing process, such as fibroblasts, osteoblasts, endothelial cells, and keratinocytes $[25,26]$. Depending on the nature of stimuli they encounter and in response to different microenvironmental factors, macrophages are "activated" and acquire a spectrum of phenotypes [27]. Macrophages at either ends of such a spectrum are commonly referred to as M1 and M2 subsets. M1 macrophages, also referred to as classically activated macrophages, are activated by IFN- $\gamma$, LPS (Lipopolysaccharide), and TNF $\alpha$. They exhibit a pro-inflammatory expression profile displayed during the early stages of the normal tissue repair process. At a later time, activated by IL-4 and IL-13, macrophages at the site of injury assume an M2 phenotype [28]. M2 macrophages, also known as alternatively activated macrophages, display an anti-inflammatory expression profile. M1 and M2 phenotypes each show specific features, such as different cell surface markers; expression of particular genes; and secretion of specific cytokines, chemokines, and enzymes (Figure 1A; [29]). 
The concept:

Fine control of the M1-M2 equilibrium using controlled release strategies
A

Nanoparticle-delivery of: siRNA anti-TNF- $\alpha$ Recruiting factors Anti-inflammatory cytokines Anti-TNF mAbs Peptides

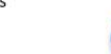

Physical cues to induce the M2 phenotype

\section{B}

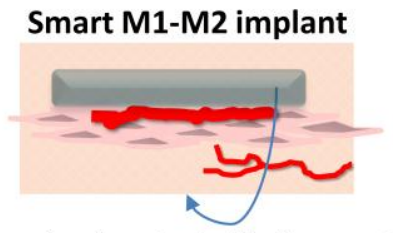

Triggering/control of inflammation, tissue healing, and vascularization

TNF- $\alpha$

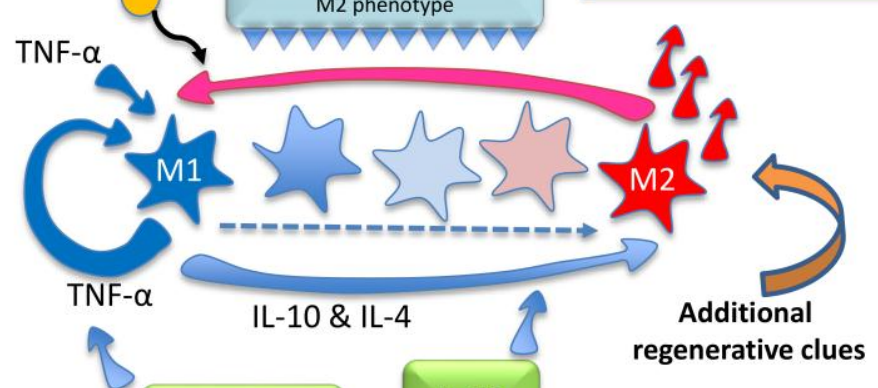

regenerative clues

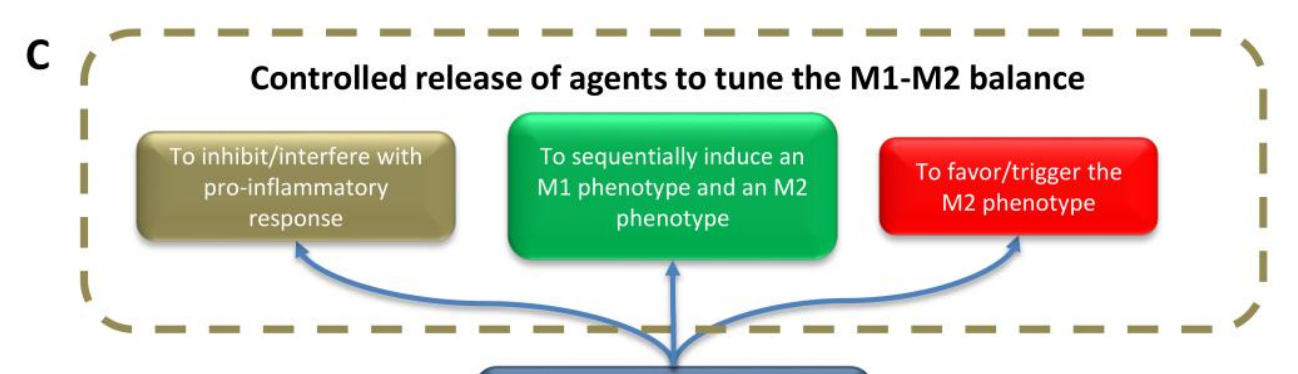

Different controlled release

strategies

to control M1-M2 balance

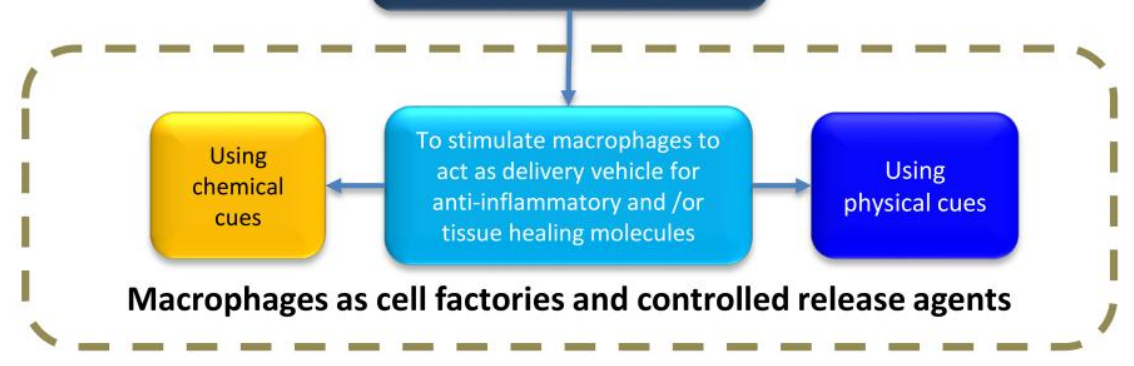

Figure 2. The progression of stages from the M1 to M2 phenotypes can be controlled at various time points through different strategies. (A) A simplified representation of the signaling network relevant to inflammation and healing after implantation of a scaffold. The main actors in the network are represented: TNF $\alpha$, IL-4, and IL-10. M1-M2 polarization can be controlled or disrupted by the controlled release of different molecules that interfere with pro-inflammatory cytokines (e.g., release of anti-TNF $\alpha$ monoclonal antibodies (mAbs) or proteins) or amplify anti-inflammatory stimuli (e.g., release of IL-10). Micro- and nanoparticles loaded with different chemical agents (e.g., siRNA, DNA, anti-TNF $\alpha$ mAbs, IL-10 and other cytokines, etc.) have been used as controlled-release agents to modulate the M1-M2 balance. (B) The ultimate aspiration would be to design and fabricate "smart tissue engineering scaffolds" 
with controlled release potential that would regulate the M1-M2 balance (and therefore the healing, tissue repair, and vascularization processes). While the M1 macrophage phenotype is needed immediately after injury, and inflammation is an important and required component of the proper response of the human body to injury, a timely shift towards the M2 phenotype is required to complete the healing/tissue regeneration process. (C) The different controlled release strategies reviewed here that aim to tune the M1-M2 equilibrium.

Arguably, IL-1 $\beta$ and TNF $\alpha$ are the two most prominent pro-inflammatory cytokines since they are overexpressed and have a role in almost every inflammatory disease [8]. The M2 phenotype has been associated with the secretion of several different cytokines and factors. The anti-inflammatory cytokines secreted by the M2 phenotype interfere with or inhibit proinflammatory cytokines secreted by the M1 type. For instance, IL-10, one of the most important anti-inflammatory cytokines and main biochemical signature of M1 macrophages, suppresses IL6 and TNF $\alpha$ production (Figure 2A; [30-33]).

Although historically, the terms "pro-inflammatory" and "pro-healing" have been associated with the M1 and M2 phenotype, respectively, the current state of knowledge in the field does not support the oversimplified notion of M1 macrophages as being detrimental for healing and M2 macrophages as being positive for healing. For example, anti-inflammatory treatments can promote healing of diabetic ulcers, but only if they are administered three days after injury [34]. On the other hand, sustained M1 activation hinders healing [35]. M1 macrophages are required for wound healing, but they must subside after a period of a few days. Moreover, M2 macrophages have long been known to cause fibrous encapsulation of biomaterials (i.e., [36]). In summary, M1 and M2 macrophages are both needed at different time points in the healing process. Promotion of a rational and timely control of the M1-M2 balance throughout the healing process seems to be key in tissue engineering and regenerative medicine applications. 
Recent work now challenges the traditional M1 and M2 framework (see for example $[8,27,31,37,38])$. Increasingly, the $\mathbf{M 1}$ and $\mathbf{M} 2$ phenotypes are being perceived as two extremes of a continuum of functional states; consequently, in any real biological scenario, a spectrum of macrophage subpopulations, ranging from the M1 to the M2 phenotype, will be encountered (Figure 2A). The balance of these subpopulations will then determine the biochemical environment prevalent in the local tissue [31]. Moreover, current experimental evidence suggests that each individual macrophage displays a unique set of combined markers and will produce a unique repertoire of biochemical signals that will situate it at a singular distance from the M1 and M2 extremes [31]. Recently, Xue et al. analyzed the transcriptome of human macrophages activated with different chemical stimuli including receptor ligands, cytokines, and metabolic cues [27]. The authors conducted a clustering analysis of the transcriptome signals using bioinformatics tools (Figure 1C(a)). The transcriptome signatures aligned well along an axis when macrophages were stimulated with agents recognized as M1 (IFN- $\gamma$, LPS, TNF) or M2 (IL-4, IL-13, IL-10) activation cues (Figure 1C(b-c). However, when other activators were used (e.g., free fatty acids, high density lipoprotein (HDL), or chemical cues associated with chronic inflammation) seven additional phenotypes were observed (Figure $1 \mathrm{C}(\mathrm{d})$ ). These results suggest that the M1-M2 macrophage polarization model, when understood as a spectrum along an axis between two extreme phenotypes, reasonably describes the biochemical plasticity of macrophages exposed to cues that are known to activate the M1 or M2 phenotypes. The M1-M2 model has to be expanded to a multi-axis spectral model (Figure 1C(a) to fully capture the biochemical plasticity of macrophages when exposed to a wider range of stimuli. 
The recent use of state-of-the-art transcriptomic tools has allowed the identification of key molecular players and their roles in the control of M1-M2 polarization. A good summary of the current knowledge on this matter has been presented in recent reviews [8,37]. Despite the evolving view of M1 and M2 macrophage polarization, the present review will often refer to the simplified case of two distinctive phenotypes, M1 and M2, since many studies in the literature have adhered to this paradigm and reported their results in this manner.

Researchers exploring tissue engineering applications aim to achieve a short (and yet sufficient) pro-inflammatory period in which M1 macrophages are recruited to the site, followed by an anti-inflammatory stage where the M2 phenotype dominates. Several strategies can be used to achieve this aim (Figure $2 \mathrm{~B}$ and $2 \mathrm{C}$ ); this review focuses on biomaterials-based strategies that examine (a) the controlled delivery of molecules to minimize pro-inflammatory or promote anti-inflammatory or tissue healing responses (Figure $3 \mathrm{~A}-\mathrm{C}$, (b) the targeted transfection of macrophages to overexpress anti-inflammatory genes or inhibit the expression of pro-inflammatory molecules (Figure 3D), and (c) the use of physical or mechanical cues to influence macrophage polarization in situ (Figure 4).

\section{Delivering Molecules to Control Macrophage Polarization}

Most reports currently available in the field of injury management and tissue regeneration have focused on the control of cytokine levels as a strategy to control inflammation or to promote faster healing. Not until recently have several reports discussed different strategies for modulating M1-M2 polarization.

This review will mainly discuss biomaterials-based strategies that specifically influence macrophage polarization. Most of the strategies for controlling the M1-M2 balance or for 
promoting faster healing have focused on interfering with the inflammatory effect of TNF $\alpha$ or promoting the release of IL-10 (a known anti-inflammatory agent mainly secreted by M2 macrophages). However, we will also review a wider range of controlled release strategies that aim to intervene at different pathway points in the complex network associated with inflammation and healing.

\section{Releasing antibodies against TNF $\alpha$ to mitigate inflammation}

Due to its potent pro-inflammatory properties, blocking TNF $\alpha$ is an obvious strategy for attenuating inflammation in tissue engineering applications. TNF $\alpha$ is a cytokine that mediates immune and inflammation overreactions in many pathological scenarios and is therefore an important target for anti-inflammatory drugs $[39,40]$. TNF $\alpha$ is produced by M1 macrophages and has been referred to as an inducer of the M1 phenotype [41,42].

To our knowledge, only a limited number of studies have specifically examined the effect of a local release of an anti-TNF $\alpha$ compound on M1-M2 polarization. However, TNF $\alpha$ has been reported as a therapeutic target for impaired cutaneous wound healing [43]. The topical addition of anti-TNF $\alpha$ neutralizing antibodies accelerated wound healing and altered the M1-M2 balance, shifting it towards an M2 phenotype, in a mouse model with severely impaired wound healing and excessive inflammation [44]. In addition, a number of studies have examined the controlled release of anti-TNF $\alpha$ agents for mitigation of inflammation in a context different than injury healing and some in the case of burn healing; these studies provide insight into potential strategies for mitigating inflammation and possibly tuning the M1-M2 balance in tissue engineering applications.

Many different therapeutic formulations are available commercially to block TNF $\alpha$. Among these are monoclonal antibodies (mAbs) (e.g., infliximab, adalimumab, and golimumab), 
antibody fragments (e.g., certolizumab pegol), or fusion recombinant proteins (e.g., Etanercept), which are common therapeutic choices in many autoimmune diseases such as rheumatoid arthritis (RA), mainly due to their selectivity [44]. Anti-TNFa mAbs are normally delivered systemically by intravenous injection; however, their harmful side effects when administered this way [44-46] have prompted the experimental development of local delivery strategies using controlled release systems. Several recent contributions have described the fabrication of engineered materials for the controlled release of anti-TNF $\alpha$ antibodies in different biological or physiological scenarios (see also the review by Grainger [47]). For example, Shamji et al. entrapped a commercial anti-TNF $\alpha$ mAb into chitosan gels prepared by co-dissolving with glycerophosphate [48]. They observed total and sustained in vitro release (of $8 \mu \mathrm{g}$ of mAb per $\mathrm{mL}$ of gel) over 10 days. The mass fraction of $\mathrm{mAb}$ released from the chitosan gels increased nearly linearly with time during the first 10 days.

In one recent study, anti-TNF $\alpha$ antibodies were conjugated to hyaluronic acid (HA) to mitigate inflammation in burns $[48,49]$. The authors compared equivalent doses of locally delivered $\mathrm{mAb}$ and $\mathrm{HA}$ versus the conjugated (anti-TNF $\alpha \mathrm{mAb}$ )-HA formulation in an in vivo rat wound model. The (anti-TNF $\alpha \mathrm{mAb}$ )-HA formulation was more effective in triggering earlier wound healing than an equivalent dose of locally delivered mAb. In addition, the authors observed significantly less secondary necrosis in rats treated with (anti-TNF $\alpha$ )-HA. These authors suggested that conjugation simply slowed down the diffusivity of the mAb, which then modulated the intensity of the inflammation cascade.

The use of particles to encapsulate (or attach) and release anti-TNF $\alpha$ molecules has been recently reported by different groups. In two separate studies, Foong et al.[50] and Marquette et al. [51] encapsulated a full-length anti-TNF $\alpha$ mAb into poly(lactic-co-glycolic acid) (PLGA) 
particles. Foong et al. encapsulated infliximab, a commercial anti-TNF $\alpha$ mAb, within PLGA particles using a high speed dispersion strategy combined with thermally induced phase separation (TIPS) [50]. The authors tested the neutralizing activity of the released antibody against recombinant TNF $\alpha$ in experiments where L929 fibroblasts were incubated in the presence of cytotoxic concentrations of recombinant TNF $\alpha$. Fibroblast viability was significantly higher in experiments where anti-TNF $\alpha \mathrm{mAb}$ releasing particles were used than when equivalent concentrations of soluble anti-TNF $\alpha \mathrm{mAb}$ were used. In the more recent study, Marquette et al. produced anti-TNF $\alpha$ IgG loaded microparticles (average diameter $<100 \mu \mathrm{m}$ ) by dispersing the spray-dried antibody and the PLGA particles into a solid-oil-water emulsion where ethyl acetate was used as an organic solvent [51]. The authors demonstrated that these anti-TNF $\alpha$ antibody particles retained biological activity after 4 weeks when stored at $5{ }^{\circ} \mathrm{C}$. In vitro release profiles showed that this delivery system slowly released 40 to $80 \%$ of the loaded anti-TNF $\alpha$ antibody over a time window of 4 weeks. Carrillo-Conde et al. used poly-anhydride nanoparticles for the delivery of active anti-TNF $\alpha$ monoclonal antibodies [52]. Using a very extensive set of in vitro and in vivo (mouse model) assays, the authors demonstrated that these nanoparticles preserved the biological activity and functionality of the antibody and provided an effective vehicle to control its release kinetics for relatively extended periods of time (up to 20-30 days).

In yet another study, Etanercept, a commercial anti-TNF $\alpha$ fusion protein of the TNF $\alpha$ receptor and the constant portion of the IgG1 antibody, was encapsulated in microspheres fabricated with poly(e-caprolactone) (PCL) and its co-polymer with poly(ethylene glycol), methoxy-poly(ethylene glycol)-poly(e-caprolactone)-methoxypoly(ethylene glycol) (MPEGPCL-MPEG) [53]. Sustained Etanercept release was observed in in vitro experiments using fibroblast-like synoviocyte cells. A significant decrease in levels of pro-inflammatory cytokines (namely interferon gamma (IFN $\gamma), \quad$ TNF $\alpha, \quad$ IL-6, and IL-17) and matrix 13 
metalloproteinases (MMPs, namely MMP-3 and MMP-13) was observed in the synoviocyte cell cultures. These cells are important players in maintaining homeostasis at the inner layer (intima) of the synovium, or the inner lining of joints. Fibroblast-like synoviocytes also have an important role in rheumatoid arthritis (RA). Synovial hyperplasia (an over-proliferation of these cells) is a typical feature of RA; fibroblast-like synoviocytes also release a number of pro-inflammatory signal molecules, especially IL-6, IL-8, prostanoids, and MMPs, which potentially affect other cells and also enhance inflammation [54].

More recently, Wang et al. presented a glucose-sensitive system capable of local longterm mitigation of inflammation by delivering an anti-TNF $\alpha$ antibody through a chitosan and collagen scaffold [55]. This collagen-chitosan scaffold was capable of attenuating the inflammatory response to bone marrow stromal cells under a condition of hyper glucose and TNF $\alpha$. In addition, the authors investigated the biological effects of the scaffold in a diabetic rat model with an ample fluctuation of blood glucose levels. An enhanced expression of osteogenic proteins and alveolar bone healing was achieved in this diabetic rat model.

The controlled release of anti-TNF $\alpha$ mAbs has been commonly reported in the context of inflammatory diseases but has yet to be assayed in the specific context of M1-M2 regulation. The use of anti-TNF mAbs alone or in combination with other anti-inflammatory molecules could be a valuable addition to the toolbox currently available to attenuate local inflammation and promote faster tissue healing.

\section{Release of cytokines to control M1-M2 dynamics}

Restoration of the normal cytokine equilibrium at a specific site of inflammation is another obvious strategy for inhibiting inflammation and promoting healing [56]. Among the multiple alternatives available for restoring cytokine homeostasis, manipulation of the local 
concentrations of IL-10, an anti-inflammatory cytokine, has been widely used. For instance, Carvalho et al. produced a biologically active recombinant mutant version of IL-10 in Escherichia coli cultures and showed that this cytokine was spontaneously incorporated into a dextrin nanogel matrix [57]. The resulting complex stabilized the protein and allowed its slow release (up to $15 \%$ during the first few hours). One challenge with using IL-10 is that it is effective at reducing inflammation in animal models but has not yielded equally successful results in human clinical trials [58].

The sequential release of chemical signals has been recognized as an important part of the delicate and complex system by which tissues undergo auto-repair. The design of scaffolds capable of sequential release of healing factors or molecules has been proposed and tested by several groups. Kumar et al. (2015) have recently described the use of a biomimetic multidomain peptide hydrogel capable of sequestering cytokines in its nanofibrous matrix [59]. The material was engineered to achieve a biphasic pattern of cytokine release to activate monocytes and macrophages using monocyte chemotactic protein (MCP-1) and IL-4. Furthermore, macrophage-material interactions were promoted without generation of a pro-inflammatory environment. Macrophage interaction with and response to the peptide composite facilitated (i) the recruitment of monocytes/macrophages, (ii) a sustained residence of immune cells until degradation, and (iii) promotion of a pro-resolution M2 environment.

Recently, Spiller et al. modified decellularized bone to release IFN $\gamma$ at early times to promote the M1 phenotype, which secretes VEGF and is thus involved in initiating the process of angiogenesis, followed by a more sustained release of IL-4 to promote the M2 phenotype, which secretes PDGF-BB and is thus involved in later stages of angiogenesis for bone regeneration [28]. Different strategies, including physical adsorption and biotin-streptavidin 
affinity binding, were used to respectively bind IFN $\gamma$ and IL-4 to the scaffolds. IFN $\gamma$ was released over the first three days, whereas most IL-4 was released during the first six days. These decellularized bone scaffolds effectively triggered sequential polarization from a dominant M1 to a dominant M2 phenotype as measured by the secretion of four cytokines and determination of ten M1 or M2 molecular markers. However, the overlap between the phases of IFN $\gamma$ and IL-4 release masked a more defined polarization. Finally, a murine subcutaneous implantation model showed increased vascularization in bone scaffolds releasing IFN $\gamma$ and IL-4 as compared to plain scaffolds.

The sequential release of molecules is inspired by the natural healing process of tissue. The development of sequential release strategies (and the engineering of materials capable of doing so) promises to significantly advance the state of the art in M1-M2 modulation in tissue engineering and will therefore be an important research topic in the near future.

\section{Release of other anti-inflammatory molecules}

Besides biologicals, several other compounds also exhibit anti-inflammatory properties. These immune-modulatory molecules range in chemical complexity and origin from proteins isolated from diverse sources to small molecules isolated from plants. For example, triptolide is a trypanosome-suppressive immunomodulating factor (TSIF) [60] that is a widely used medicinal compound isolated from the Chinese herb Tripterygium wilfordii. It has been released from gelatin hydrogels in combination with BMP-2 (bone morphogenetic protein-2) to accelerate bone repair [61]. In this section, we examine the use of some of these anti-inflammatory molecules and emphasize those that have been used in controlled release strategies in tissue engineering or tissue repair scenarios. 
In addition to cytokines, studies have introduced other molecules to manipulate the M1M2 response. For example, coating collagen scaffolds with bacterial lipopolysaccharide (LPS) caused an increase in blood vessel infiltration and was accompanied by high levels of M1 and M2 macrophages [62].

Roh et al. demonstrated that vascularization of poly-L-lactide and poly- $\varepsilon$-caprolactone $[\mathrm{P}(\mathrm{CL} / \mathrm{LA})]$ scaffolds was strongly dependent on the action of recruited macrophages and was enhanced by the delivery of MCP-1 using alginate microparticles [63]. In another study, FTY720, a sphingosine 1-P (S1P) receptor agonist was released from poly(lactic-co-glycolic acid) thin films in inflamed and ischemic tissues [64]. This delivery resulted in a reduction in proinflammatory cytokine secretion, an increase in anti-inflammatory cytokine secretion, and increased vascularization in rodents. Similarly, Das et al. reported that local delivery of FTY720 from composite poly(DL-lactide-co-glycolide) (PLGA) nanofibers enhanced blood vessel growth, increased M2 macrophage recruitment, and led to significant bone tissue proliferation within critical-size bone defects after 12 weeks of treatment [65]. Kim et al. studied the effect of two agents, the macrophage recruiting agent SEW2871 (a sphingosine-1 phosphate receptor agonist) and platelet-rich plasma (PRP), on bone remodeling when applied alone or in combination [66]. The authors formed micelles of SEW2871 (non-water soluble) in gelatin grafted with L-lactic acid. These micelles and PRP were then incorporated into gelatin hydrogels, which allowed the controlled release of SEW2871 and PRP. These molecules acted synergistically to enhance macrophage migration in in vitro assays. In experiments conducted in a rat bone defect model, a higher number of macrophages was recruited by mixed hydrogels containing both SEW2871 micelles and PRP than by hydrogels containing only one of the components. In addition, the use of this combined released system appeared to promote a sequential induction of inflammatory 
responses. Three days after application, an enhanced level of TNF $\alpha$ was observed. Later, an increase in the expression of osteoprotegerin (OPG) and IL-10 was observed.

A

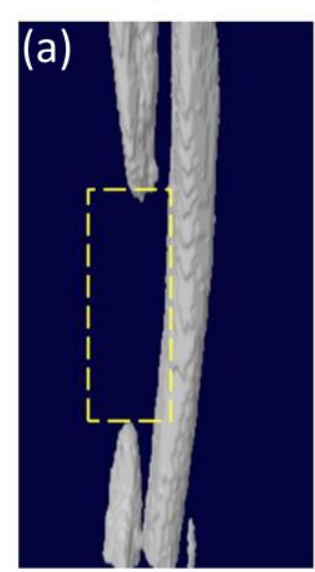

B

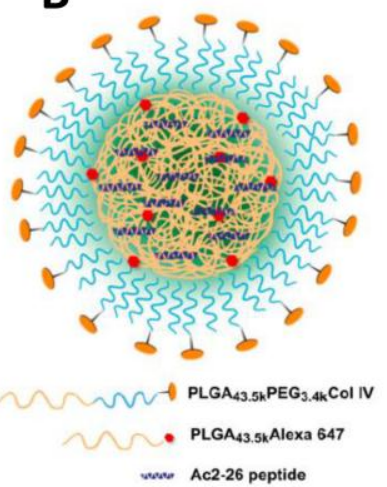

D

(a)
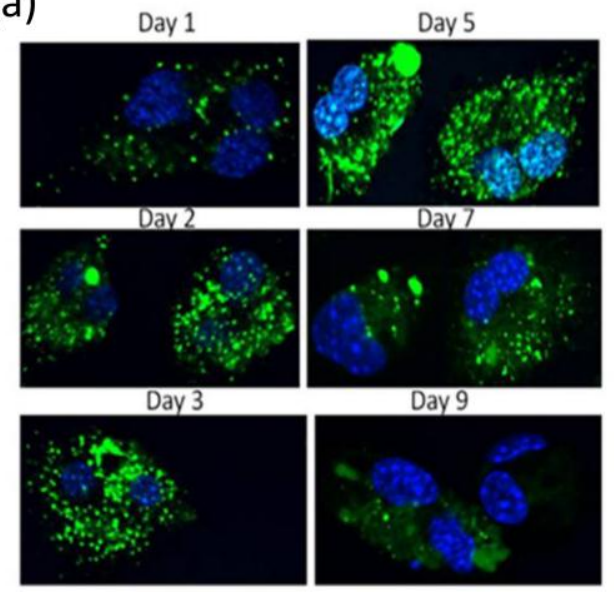

C
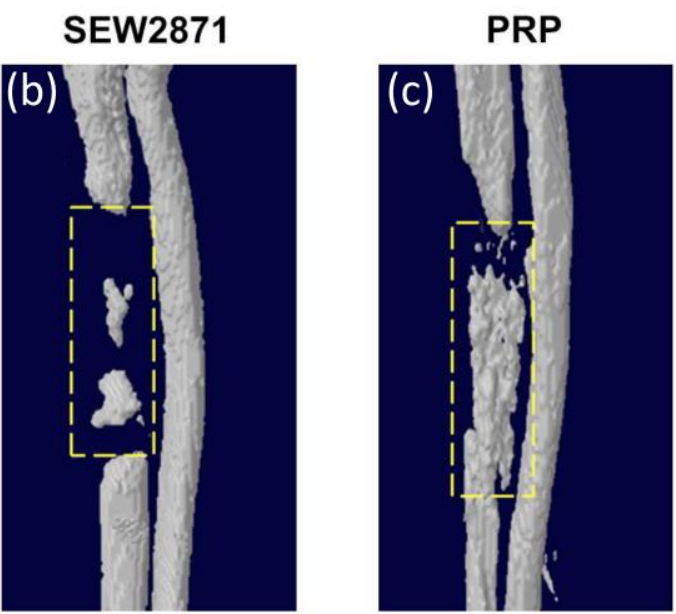

SEW2871/PRP
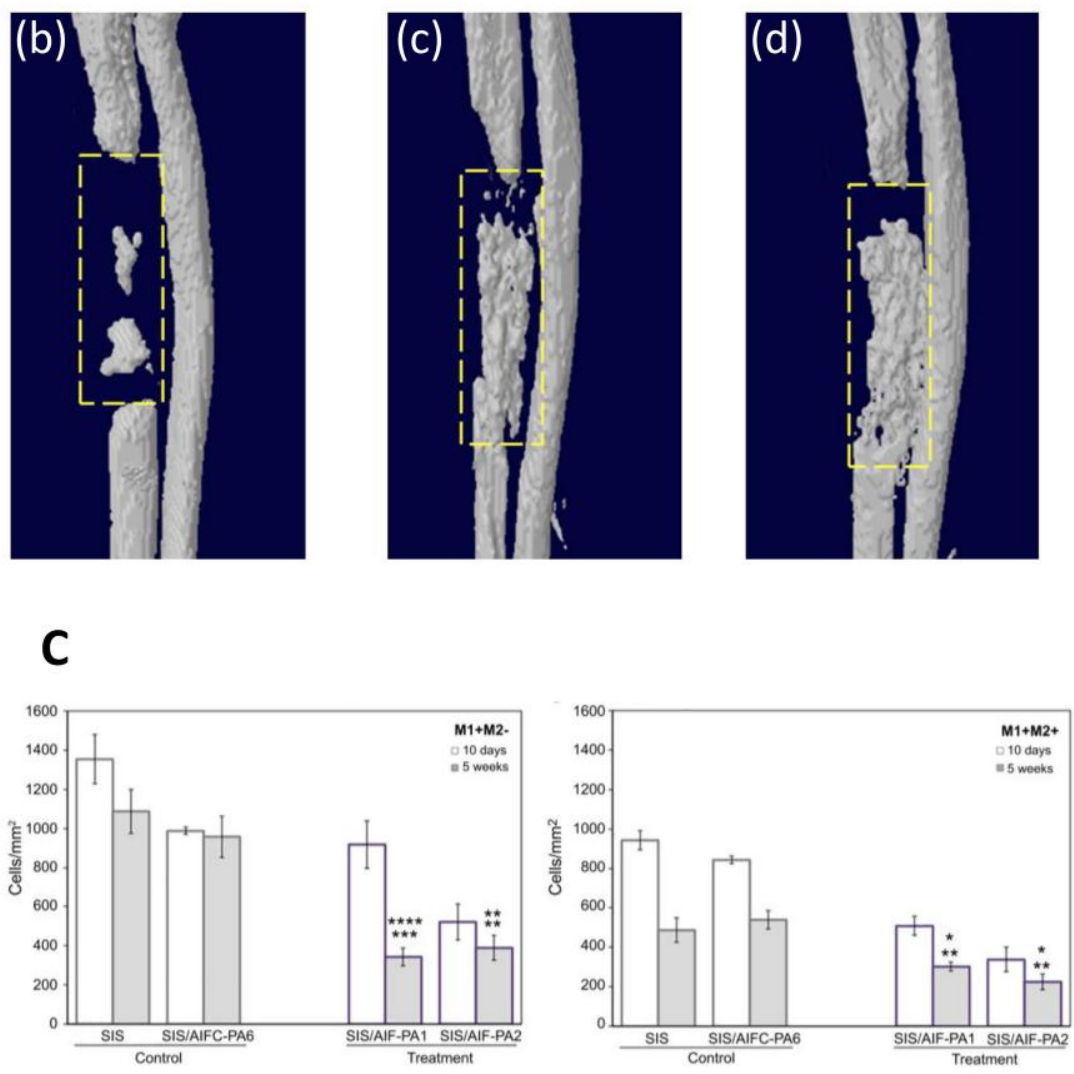

(b)

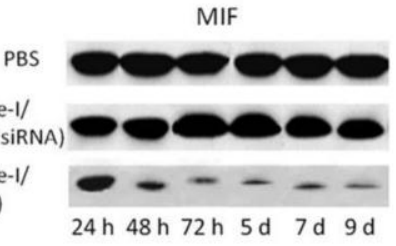

(c)

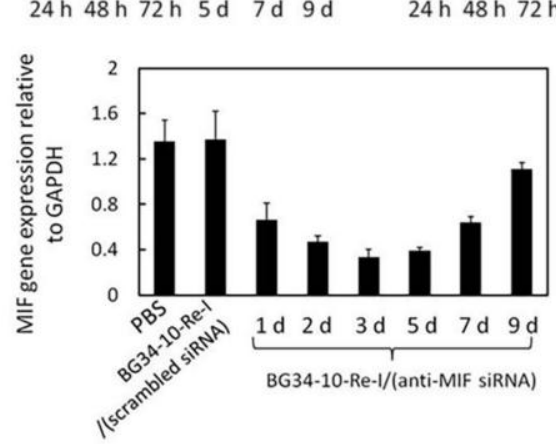

BG34-10-Re-I/ (scrambled siRN BG34-10-Re-1/ (MIF SIRNA)
B-actin

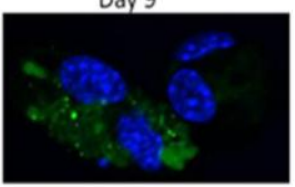


Figure 3. Examples of controlled release strategies used to modulate the M1-M2 balance in tissue engineering applications. A) Use of a hydrogel scaffold loaded with immunomodulators. Threedimensional microCT images of bone regeneration in a rat defect model six weeks after implantation of hydrogels loaded with (a) PBS, (b) SEW281, (c) PRP, and (d) SEW287 and PRP are shown. Reprinted with permission from Kim et al., 2014 [66]. (B) Encapsulation of N-terminal peptide consisting of amino acids 2-26 of Annexin A1 (Ac2-26) in a PLGA-PEG nanoparticle conjugated to a peptide targeting collagen IV. Reprinted with permission from Kamaly et al. (2013) [75]. (C) Decellularized small intestinal submucosa (SIS) was coated with anti-inflammatory peptide amphiphiles (AIF-PAs) derived from uteroglobin protein sequences. Five weeks after implantation in a rat bladder augmentation model, SIS coated with peptides 1 or 2 (SIS/AIF-PA1/2) showed decreased numbers of M1 macrophages (labeled as M1+M2- where CD86+ cells were labeled as M1+) compared to uncoated (SIS) scaffolds or scaffolds coated with a control peptide amphiphile (SIS/AIFC-PA6). After five weeks, SIS/AIF-PA1/2 scaffolds had lower total numbers of M1 and M2 cells (M1+M2+ where CD206+ were labeled as M2+) compared to control scaffolds. Top and bottom row of asterisks represent comparisons to SIS and SIS/AIFC-PA6, respectively, and * represents $p<0.05$, ** represents $p<0.01$, *** represents $p<0.001$, and $* * * *$ represents $p<0.0001$. Reprinted from Bury et al. (2014), with permission from Elsevier [81]. (D) Delivery of AF488-siRNA and MIF expression within primary macrophages. (a) Confocal microscopy images of macrophage cultures. Nuclei were stained with DAPI (blue), and nanoparticles with siRNA were dyed green. (b) Western blots showing MIF and $\beta$-actin (used for normalization) protein expression in macrophages. (c) qRT-PCR analysis of MIF gene expression in macrophages. PBS and scrambled siRNA served as negative controls. Reprinted with permission from Zhang et al., 2015 [89].

Increased levels of TGF- $\beta 1$, another anti-inflammatory cytokine (with reported tissue fibrosis effects [67]), were also measured ten days after the intervention. A significantly greater amount of bone regeneration was induced by the hydrogels that contained a mixture of SEW2871micelles and PRP than by hydrogels that contained only one of these components or only PBS (see Figure 3A).

Vasconcelos et al. (2015) evaluated the effect of lipoxin A4 (LxA4) and resolvin D1 (RvD1), two different pro-resolution lipid mediators, on the modulation of the inflammatory response to chitosan-based scaffolds [68]. The authors conducted in vivo experiments using a mouse airpouch model of inflammation. They demonstrated that both molecules shifted the M1 macrophage inflammatory response to an M2 reparative response and decreased several proinflammatory cytokines. Recently, the same group developed chitosan-based 3D porous scaffolds loaded with RvD1 by dispersing RvD1 over freeze-dried scaffolds followed by a 
second freeze-drying step [69]. The authors investigated the inflammatory response caused by this biomaterial in an in vivo model (the mouse air-pouch model of inflammation) and found a significant shift towards an M2 macrophage population and a decrease in the inflammatory cells around and within the implanted scaffolds.

The release of anti-inflammatory agents from particles or scaffolds is an effective strategy to control inflammation in tissue engineering applications. More research is needed to fully assess the clinical potential of this approach. The spectrum of anti-inflammatory molecules that are potentially useful in tissue engineering applications range from proteins to small molecules. Among them, anti-inflammatory peptides have recently been the focus of intense research and merit a separate discussion.

\section{Controlled delivery of anti-inflammatory peptides}

Peptides offer an attractive strategy for influencing the inflammatory and healing response. Because of their shorter length, peptides are less expensive to manufacture and do not require additional processing steps (e.g., re-folding steps) compared to full-length proteins. Recent research has investigated a number of different peptides and delivery methods for reducing inflammation and introducing a pro-resolution response.

One peptide that has been studied extensively in numerous applications is $\alpha$-melanocyte stimulating hormone ( $\alpha-\mathrm{MSH})$, which is a tridecapeptide with anti-inflammatory properties. The Bellamkonda group successfully attached $\alpha$-MSH to the silicon surface of neural implants and, compared to control electrodes, observed reduced inflammation in vivo as evidenced by lower numbers of activated microglia/macrophages and reduced TNF $\alpha$ secretion [70].

Benkirane-Jessel and coworkers directly coupled the N-terminus of the $\alpha$-MSH peptide to poly(L-glutamic acid) (PGA- $\alpha-\mathrm{MSH}$ ) and fabricated polyelectroyte multilayers using 
alternating layers of PGA- $\alpha-\mathrm{MSH}$ and poly(L-lysine) (PLL) [71]. Human monocytic THP-1 cells cultured on the films had decreased TNF $\alpha$ and increased IL-10 secretion compared to cells cultured on control surfaces. Further studies showed that using these polyelectrolyte multilayers with $\alpha-\mathrm{MSH}$ to coat tracheal prostheses made of titanium beads resulted in increased IL-10 secretion compared to control prostheses [72].

Recent studies by the O'Connor group have physically adsorbed $\alpha$-MSH onto PLGA microspheres, which resulted in decreased TNF $\alpha$ production by LPS-activated RAW 264.7 macrophages at 24 and $48 \mathrm{~h}$ but not $72 \mathrm{~h}$ compared to control surfaces [73]. There was not a strong effect of the adsorbed $\alpha$-MSH when implanted subcutaneously into the backs of rats. The short-term in vitro effects and weak in vivo effects were hypothesized to be due to the rapid release of $\alpha-\mathrm{MSH}$. Thus, O'Connor and co-workers modified their system to prolong the release of $\alpha$-MSH by decreasing the diffusion rate [74]. Specifically, they made porous PLGA microspheres, physically adsorbed $\alpha-\mathrm{MSH}$, and also added polyelectrolyte layers with embedded fibroblast growth factor (FGF) on top of the $\alpha-\mathrm{MSH}$. When implanted subcutaneously, the porous microspheres that had a polyelectrolyte layer on top of the adsorbed $\alpha$-MSH had statistically fewer ED1-positive macrophages compared to microspheres with adsorbed $\alpha$-MSH and no polyelectrolyte layer. However, the untreated microspheres resulted in high variability, and there was no statistical differences between the control and treated scaffolds. Collectively, these studies demonstrate that controlled delivery of $\alpha$-MSH peptides can reduce inflammation in diverse applications.

Annexin A1 is another anti-inflammatory and pro-resolution molecule. Kamaly and coworkers encapsulated an N-terminal peptide consisting of amino acids 2-26 of Annexin A1 (Ac2-26) in a PLGA-PEG nanoparticle conjugated to a peptide targeting collagen IV (Figure 
3B), which is found in vascular basement membrane [75]. These nanoparticles reduced acute inflammation in mouse models of periotonitis and hind-limb ischemia. In addition, these polymeric nanoparticles decreased chronic inflammation in a mouse model of atherosclerosis [76]. Moreover, these nanoparticles increased healing in mouse models of colitis or colonic wounds introduced through biopsy [77]. Thus, the Ac2-26 peptide derived from Annexin A1 has strong pro-resolution effects in vivo; however, further studies need to be performed to elucidate its role in macrophage polarization. One recent study found that full-length Annexin A1 increased IL-10 secretion and decreased the M1 macrophage phenotype [78] whereas another study found that Annexin A1 did not appear to contribute to macrophage polarization [79].

Zachman and coworkers used porous scaffolds and filled the pores with collagen gels loaded with a tetrameric anti-inflammatory peptide derived from thymosin $\beta-4$ (SDKP) and/or a pro-angiogenic peptide derived from laminin (C16) [80]. In co-culture experiments with human umbilical vein endothelial cells (HUVECs) and monocyte-derived macrophages (MDMs) activated with LPS, scaffolds with SDKP alone or SDKP and C16 resulted in lower phagocytic activity and decreased levels of anti-inflammatory cytokines (IL-1 $\beta$, IL-6, IL-8, and TNF $\alpha$ ) compared to control scaffolds without peptide. When the scaffolds were implanted subcutaneously into mice, scaffolds with the SDKP peptide had lower numbers of macrophages and decreased phagocytic activity compared to scaffolds without the SDKP peptide. The SDKP peptide thus has the ability to reduce inflammation in soft tissue models.

Bury et al. recently incorporated short sequences from the uteroglobin protein into selfassembling peptide amphiphiles [81]. The peptide amphiphiles were then used to coat decellularized small intestinal submucosa (SIS), a material that has shown promise in many tissue engineering applications but is pro-inflammatory. The materials were then implanted in athymic rats in a bladder augmentation model. After ten days, rats implanted with two of the 
peptides had decreased pro-inflammatory markers (IL-1 $\beta$ and $\mathrm{TNF} \alpha$ ) and increased antiinflammatory markers (IL-10 and IL-13) compared to rats implanted with uncoated controls or a sequence-scrambled control peptide. Furthermore, after five weeks, these two peptides reduced the level of M1 macrophages compared to control scaffolds (Figure 3C). Improvements in the inflammatory status also correlated with increased bladder function. Thus, this study demonstrated the promise of these uteroglobin peptides in reducing inflammation and increasing function in a bladder tissue engineering application.

Anti-inflammatory peptides could be a more cost-effective strategy to control inflammation than full-length proteins - it is easier to chemically conjugate them to scaffolds or nanoparticles, they can be produced easily and at a relatively low cost, and they are more stable than proteins. These studies demonstrate the promise of using antiinflammatory peptides in treating inflammation. In particular, the peptides were able to modulate the secretion of pro-inflammatory and anti-inflammatory cytokines. In some cases, peptides modulated the M1-M2 balance; however, more studies are needed in this area to elucidate the effect of individual peptides on macrophage polarization.

\section{Peptides to reduce the effects of inflammatory cytokines}

The previous approaches have successfully delivered anti-inflammatory peptides to the site of interest. An alternative approach is to design scaffolds that inhibit the detrimental effects of inflammatory cytokines. Because of their small size, it is often not feasible to design a scaffold that excludes these molecules. Instead, the Anseth group designed a PEG hydrogel conjugated to the WP9QY peptide, which binds the pro-inflammatory cytokine TNFa [82]. High levels of TNF $\alpha$ induce apoptosis in many cell types. Encapsulating differentiated PC12 cells, mouse pancreatic islets, and human mesenchymal stem cells (hMSCs) in PEG hydrogels with the 
WP9QY peptide reduced the number of cells undergoing apoptosis when challenged with TNF $\alpha$ compared to cells encapsulated in control PEG hydrogels. In addition, when challenged with $\mathrm{TNF} \alpha$, islet cells maintained their levels of insulin secretion and hMSCs maintained their ability to undergo osteogenic differentiation when encapsulated in PEG hydrogels with the WPQ9Y peptide, whereas cells in control hydrogels experienced decreases in their functional abilities. This study thus showed the potential of incorporating peptides to sequester pro-inflammatory cytokines within the gel and prevent the harmful effects of pro-inflammatory cytokines.

The Messersmith group also designed gels intended to protect cells from the detrimental effects of anti-inflammatory cytokines [83]. PEG hydrogels were functionalized with an IL-1 receptor inhibitor peptide (IL-1RIP) and/or the RGD cell adhesion peptide. IL-1RIP interacts directly with the IL-1 receptor of encapsulated cells and prevents the detrimental interaction of pro-inflammatory cytokines with cells. The RGD cell adhesion peptide was shown to be important for survival of the mouse insulinoma (MIN6) cells used in this study. When cells were challenged with a cocktail of IL-1 $\beta, \mathrm{TNF} \alpha$, and IFN- $\gamma$, they had increased viability in gels with both peptides compared to control hydrogels without peptide or with a single peptide. Thus, this study demonstrated that localized delivery of peptides to cell receptors decreased the negative effects of pro-inflammatory cytokines.

These two studies demonstrate the use of peptides to mitigate the detrimental effects of inflammatory cytokines. In one case, the inflammatory cytokines were sequestered within a hydrogel, and in the other study, the cells were unable to effectively interact with inflammatory cytokines. These alternative approaches of using peptides that counteract inflammatory cytokines will continue to be an intense focus of research in tissue engineering in the following years. 


\section{Release of nucleic acids to control M1-M2 polarization}

Small interfering RNA (siRNA)and microRNA (miRNA) platforms have been proposed to regulate the immune response to implants, promote wound healing, and improve host-implant integration [84]. In particular, a limited number of studies have explored the controlled release of nucleic acids (e.g., genes, miRNAs, and siRNAs) to alleviate inflammation or promote healing by targeting macrophage-related functions in tissue engineering applications [84]. However, in other inflammatory scenarios, such as inflammatory diseases, autoimmune diseases, and cancer, the delivery of nucleic acids has been frequently tested as a means of controlling inflammation or promoting healing. For instance, several conventional gene transfer approaches for treatment of inflammatory bowel disease (IBD) have been extensively reviewed elsewhere [85]. The main aims have been to overexpress IL-10 or to block TNF $\alpha$ to control inflammation.

The delivery of genes encoding IL-10 by viral and non-viral vectors is one strategy for delivery of anti-inflammatory cytokines. Bhavsar and Amiji developed a nanoparticle-inmicrosphere oral system (NiMOS) for the controlled release of the IL-10 gene in a colitis Balb/c mouse model, which was induced using tri-nitro-benzene-sulfonic acid (TNBS) [86]. They found that IL-10 gene expression could successfully suppress the expression of several proinflammatory cytokines, including IFN $\gamma, \mathrm{TNF} \alpha$, IL-1 $\alpha$, IL-1 $\beta$, and IL-12. In addition, the animals showed clinical improvement. NiMOS technology was later used to deliver siRNA and silence TNF $\alpha$ expression in an acute colitis mouse model (induced by dextran sulfate sodium) [87]. The use of NiMOS in this experimental in vivo model also downregulated the production of IFN $\gamma$, IL-1 $\beta$, and MCP-1 and reduced myeloperoxidase activity. Furthermore, the authors 
showed that releasing both anti-TNF $\alpha$ and anti-cyclin-D1 siRNA had a stronger effect than anti$\mathrm{TNF} \alpha$ therapy alone.

Recently, Boehler et al. transfected pre-polarized macrophages with a lentivirus encoding IL-10 and measured the levels of TNF $\alpha$ produced [88]. After an inflammatory challenge, transfected cells produced significantly less TNF $\alpha$ than cells treated with either an empty control virus or a bolus dosage of recombinant IL-10 protein. These results demonstrate the technical feasibility of lentiviral transfection of IL-10 to macrophages and the potential of this delivery strategy for inducing and sustaining M2 macrophage polarization in biomedical applications.

The targeted delivery of siRNA to macrophages presents some technical challenges. Most siRNA delivery strategies rely on internalization of siRNA into dividing cells. Therefore, macrophage delivery systems have to be engineered to promote the active uptake of siRNA. Additionally, macrophages are phagocytes that produce a wide range of degradative enzymes. Any delivery vehicle that can successfully target macrophages also has to protect the siRNA against enzymatic degradation by the macrophages. Ideally, a macrophage-targeted delivery system should enable a controlled and long lasting delivery of siRNA for effective modulation of the immune response. Zhang et al. (2015) recently described a glucan-based nanoparticle carrier system to deliver siRNA (referred to by the authors as BG34-10-Re-I) [89]. These BG34-10-ReI/siRNA nanoparticles effectively inhibited the expression of macrophage migration inhibitory factor (MIF) in primary macrophage cultures both at the mRNA and protein levels (Figure 3D).

The use of virus-like particles, recognized as potent effectors of the immune system, has been also proposed as a delivery platform for immunomodulatory compounds [90]. Wilson et al. reported the use of orally delivered thioketal nanoparticles (TKNs) loaded with anti-TNF $\alpha$ siRNA for the treatment of an intestinal inflammation murine colitis model [91]. The TKNs were 
fabricated from poly-(1,4-phenyleneacetone di-methylene-thioketal), a polymer that degrades selectively in the presence of reactive oxygen species (ROS). Since high levels of ROS are characteristic of sites of intestinal inflammation, TKNs allowed the targeted release of anti-TNF $\alpha$ siRNA to tissue with inflammation. This therapeutic approach diminished the levels of TNF $\alpha$ mRNA in the colon and effectively protected animals from ulcerative colitis. Similarly, Huang et al. recently reported the use of cationic konjacglucomannan (cKGM) microparticles to release an antisense TNF $\alpha$ oligonucleotide to target intestinal inflammation in a rat IBD model [92].

Other vehicles have been proposed to deliver anti-TNF $\alpha$ siRNAs to monocytes. Examples include a new acid-degradable poly(ketal amidoamine) (PKKA) carrier, which was successfully tested in an acetaminophen-induced liver failure rat model [93], and polyamidoamine (PAMAM) dendrimers and dextran nanogels, which were assayed in vitro in LPS-activated RAW 264.7 macrophages with promising results [94].

Other siRNAs could be important targets (or agents) for modulation of M1-M2 polarization. For example, miR-21 has become an attractive subject of study in this context. MiR-21 appears to play a dynamic role in inflammatory responses by acting as a mediator of the balance between the M1 and M2 phenotypes and the transition between the pro-inflammatory and antiinflammatory states [95]. The induction of miR-21 has been described as a "molecular rheostat" that regulates the inflammatory balance. However, more research is needed to fully identify all miR-21 mRNA targets and the signaling pathways and cellular processes that it regulates [95].

The controlled release of nucleic acids holds great promise to finely and specifically control the dynamics of the M1-M2 balance. However, this approach faces important challenges, which are mainly related to safety concerns and stability in in vivo settings. More fundamental and 
translational research needs to be performed in the near future to address these concerns and clear the path for these technologies in clinical applications.

\section{Biomaterial Strategies to Manipulate M1 vs. M2 Differentiation}

Several recent reports show that macrophage polarization into M1 or M2 phenotypes can be regulated not only by chemical cues (e.g., soluble factors), but also by physical cues present in the extracellular environment (Figure 4).

These findings are especially attractive in the context of tissue engineering because they open up the possibility of modulating the immune response to a foreign material (implant) by simply manipulating the material's physical properties. Several reports state that the cellular shape, expressed markers, and cytokine or chemokine secretion profiles of macrophages can be manipulated by controlling surface patterns [32,96], surface roughness [97], feature sizes (e.g., micro- or nano-topographies) [98], porosities [96,99], and substrate stiffness [100] or by culturing in 2D or 3D scaffolds [101,102]. Rostam et al. present a comprehensive table that summarizes the results of several studies on the effects of surface topographies on macrophage behavior [103].

The notion that physical cues have an effect on macrophage behavior may explain the fact that M1 and M2 macrophages can co-exist in the very same locations-where the chemical environment is practically the same-and still exhibit different secretion profiles and phenotypes. Small differences in the local topography may exist and induce different macrophage phenotypes [32]. 
A

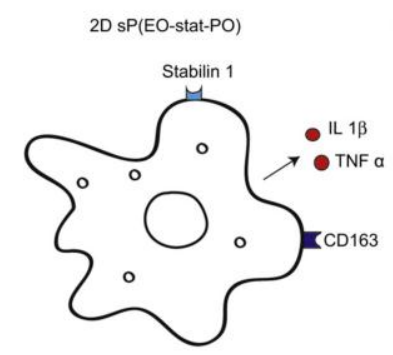

3D sP (EO-stat-PO) coated nanofibres

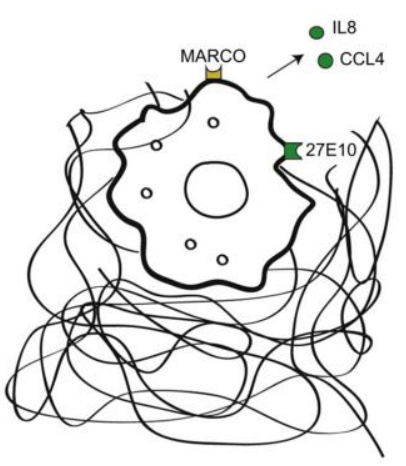

B
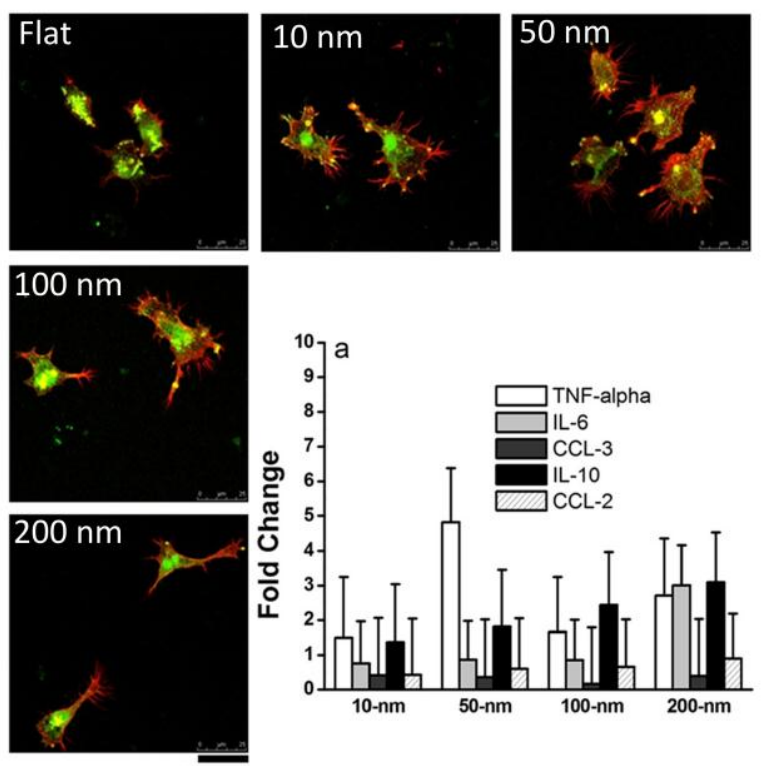

$25 \mu \mathrm{m}$

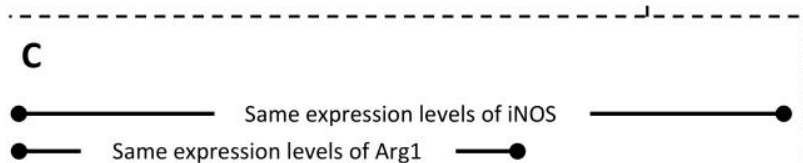

$E$
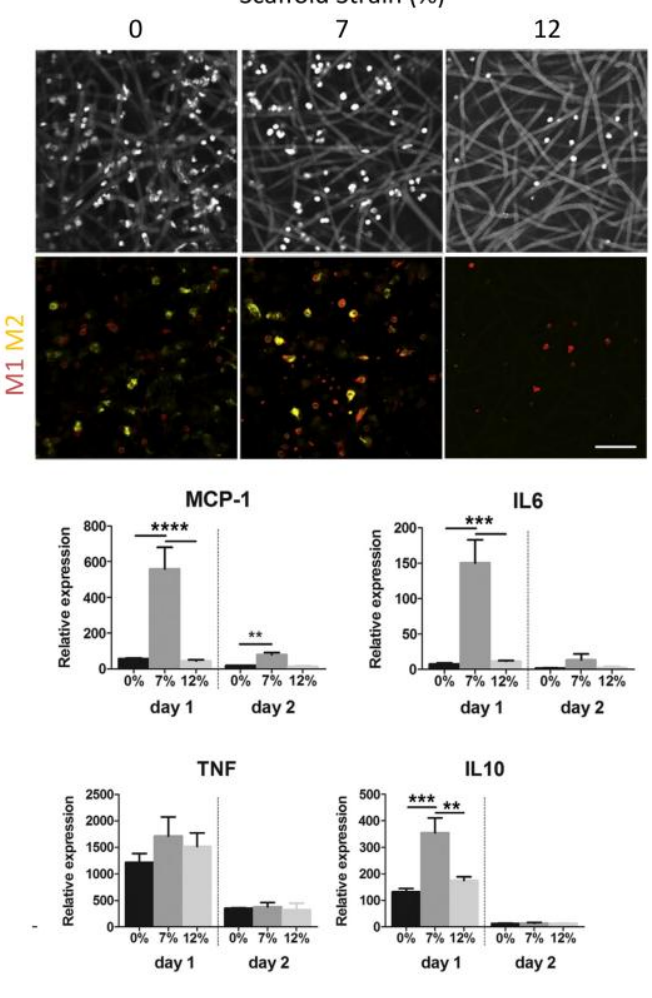

Trend of iNOS

expression

Trend of Arg1
expression

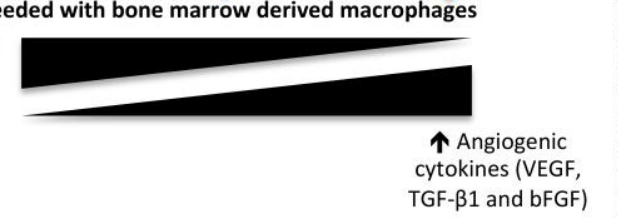

Figure 4. Effect of physical cues found in different scaffolds. (A) Cartoon showing the effect of culturing macrophages in $2 \mathrm{D}$ vs. 3D substrates of the same chemical composition. Reprinted with permission from Bartneck et al., 2012 [101]. (B) Macrophages seeded in scaffolds with nanotopographies composed of nanodots on the order of 10-200 nm. Micrographs show the effect on cell shape, and 
histograms show the effect on the cytokine and chemokine gene expression profile. Reprinted with permission from Mohiuddin et al., 2012 [98]. (C) Micropatterned scaffolds promote cell elongation. The induced cell shape derives from expression of differential markers and secretion of several cytokines. Reprinted with permission from McWhorter et al., 2013 [32]. (D) Electrospun sheets containing various polymer concentrations form scaffolds with different porosities and surface area. Macrophages respond differently to each scaffold architecture and show M2-like behavior when seeded on scaffolds produced with high concentrations of polymer. Reprinted with permission from Garg et al., 2013 [102]. (E) Macrophages adhering to electrospun scaffolds and subjected to different strain levels. The applied deformation affected the macrophage phenotype and gene expression profile. Reprinted with permission from Ballota et al., 2014 [109].

In the framework of tissue engineering, a promising strategy would be to design

\section{biomaterials capable of inducing the M2 phenotype in order to moderate the inflammatory}

host response to the foreign material. As previously mentioned, the M1-M2 classification is an evolving framework that has shifted to view the classification as a continuum. Several authors interested in the polarization of macrophages toward the M2 phenotype using physical cues have argued that the M1-M2 classification is not always accurate and sometimes can even be misleading since M1 and M2 macrophage characteristics frequently overlap. For instance, Bartneck et al. reported a comparison of macrophages cultured in 2D and 3D scaffolds with the same chemistry [101]. Their aim was to induce an M2 phenotype. Instead, they found that the 2D system yielded macrophages that exhibit not only M2 markers $\left(\mathrm{CD} 163^{+}\right)$but also an unexpected release of significant amounts of pro-inflammatory cytokines. The 3D system yielded macrophages that had M1 markers $\left(27 \mathrm{E} 10^{+}\right)$but had a dominant M2-like secretion profile (Figure 4A). Mohiuddin et al. [87] showed that size differences, even at the nanoscale, have an effect on cell attachment and expression of inflammatory genes. Macrophage attachment was higher on surfaces with 10-50 nm features than on flat surfaces and lower on 100-200 nm nanotopographies. Also, they observed that pro-inflammatory genes were mildly up-regulated on surfaces of 100-200 nm features (Figure 4B). 
The macrophage response to rough surfaces has been studied by several groups. Although these studies agree that roughness influences macrophage behavior, at present, no clear consensus has been reached on the actual effect that this physical cue has on macrophage phenotype. Lee et al. observed that macrophages cultured on nano-structured titanium surfaces showed a more moderate secretion of pro-inflammatory cytokines and reduced production of nitric oxide when compared to macrophages attached to a flat titanium surface [104]. In contrast, Refai et al. reported that more pro-inflammatory chemokines and cytokines (TNF $\alpha$, IL-1 $\beta$, IL-6, MCP-1, and macrophage inflammatory protein (MIP)-1 $\alpha$ ) were secreted by macrophages cultured on rough titanium surfaces (produced by a sandblasting and acid etching process) than by macrophages cultured on polished titanium surfaces [105]. This effect was magnified when macrophages were chemically stimulated with LPS. Likewise, Alfarsi et al. (2014) found that 16 pro-inflammation related genes were up-regulated in macrophages cultured on rough titanium surfaces when compared with macrophages cultured on polished titanium [106]. In a similar experiment, Barth et al. observed that macrophages cultured on sandblasted and acid-etched titanium surfaces exhibited a dominant M2 behavior; they displayed reduced secretion of interferon gamma-induced protein 10 (IP-10) (M1-related cytokine) and upregulated secretion of MCP-1 and MIP-1 $\alpha$ (M2-related characteristic) when compared with macrophages cultured on a polished substrate. However, these macrophages exhibited a low expression of Arg1, which is not consistent with a typical M2 phenotype [107]. The discrepancies in these studies may be due to the inherent differences of the cell lines used in each study since macrophages from different species or anatomical sites are known to behave differently [108].

Several authors have also studied the effect of micropatterned structures on macrophage functionality. McWhorter et al. reported a successful way to polarize macrophages towards an M2 phenotype by manipulating cell shape [32]. They demonstrated that the use of culture 
surfaces micropatterned with lines of $20 \mu \mathrm{m}$ width (with a $20 \mu \mathrm{m}$ distance between lines) induced macrophage elongation. In these cultures, several M2 phenotype markers were expressed, but the secretion of some anti-inflammatory cytokines was reduced (Figure 4C). Better results were obtained when macrophages were cultured on these elongation-promoting surfaces and also exposed to low doses of M2-inducing cytokines (e.g., IL-4/1L-13); elongated macrophages expressed M2 markers much more efficiently than non-elongated macrophages exposed to the same level of cytokines. In addition, the authors found that cytoskeletal contractility had an important role in the development of an M2 phenotype when induced by shape elongation. This phenomenon was demonstrated by pharmacologically inhibiting the actinmyosin contractility in macrophages cultured on the micro-patterned surfaces. Although the macrophages were successfully elongated, they did not show an upregulated expression of arginase-1, an M2 marker that had been overexpressed in analogous experiments conducted in the absence of actin-myosin inhibition.

In another study, Bartneck et al. cultured macrophages on surfaces micropatterned with different geometrical features: lines and large and small posts (widely separated and closely packed) [96]. They found that the different micropatterned shapes were able to induce important differences in the macrophage phenotype. Macrophages cultured on substrates with line patterns (10 $\mu \mathrm{m}$ width separated by $30 \mu \mathrm{m}$ ) predominantly developed surface marker profiles characteristic of M1 (high expression of 27E10 and low expression of CD163). The cells cultured on line patterns also secreted higher levels of MIP-1 $\alpha$ but lower levels of CCL2 (both pro-inflammatory chemokines) when compared with macrophages cultured on surfaces with the other micropatterns. In addition, the secretion of IL-1 $\beta$ was completely inhibited. Macrophages cultured on surfaces decorated with large posts $(20 \mu \mathrm{m}$ in diameter and separated by $70 \mu \mathrm{m})$ developed a non-inflammatory profile. In particular, they showed low expression of the 27E10 
surface marker, high expression of CD163 (M2-like phenotype), and down-regulation of the proinflammatory chemokines MCP-1 and IP-10. Macrophages cultured on lines and large post micropatterns showed an increased IL-6 secretion compared to cells attached to surfaces with small posts. However, the IL-6 concentration levels were two orders of magnitude lower than in experiments where chemokines were induced with LPS. These results suggest that physical cues trigger weaker M2 polarization responses than chemical cues. Macrophages cultured on surfaces containing small posts ( $3 \mu \mathrm{m}$ in diameter) also showed different behaviors depending on the spacing between the posts. Surfaces with a packed post array (posts separated by $6 \mu \mathrm{m}$ ) induced an enhanced secretion of MCP-1 with levels comparable to those observed by LPS induction. Greater post separation induced lower secretion levels of MCP-1. A combination of packed and separated post arrays triggered a weak secretion of IL-1 $\beta$.

Electrospinning has been also used as a technique for producing scaffolds that can provide different physical cues for macrophages in the form of different fiber thicknesses, surface areas, structural alignments (aligned or random structures), and porosities [102,109,110]. For instance, Garg et al. produced electrospun scaffolds with different architectures by using three different polymer (polydioxanone) concentrations [102]. The fiber diameter, pore size, and porosity of these scaffolds were directly proportional to the polymer concentration (Figure 4D). The expression of the Arg1 marker (characteristic of the M2 phenotype) correlated with the polymer concentration used to fabricate the scaffold; the higher the polymer concentration, the higher the expression of the Arg1 marker. Consistent with these results, expression of iNOS (an M1 marker) showed the opposite trend. In addition, macrophages on higher polymer concentration scaffolds, which expressed the Arg1 marker, also secreted higher concentrations of angiogenic cytokines (VEGF, TGF- $\beta 1$, and basic FGF) when compared to macrophages cultured 
on lower polymer concentration scaffolds. Scaffolds fabricated with similar fiber diameters but different porosities suggested that these polarization effects might be attributed mainly to the pore size (ranging from $\sim 1-15 \mu \mathrm{m}$ ) rather than to the fiber diameter (ranging from $0.35-2.3 \mu \mathrm{m}$ ).

The effect of fiber diameter was also a subject of study for Saino et al. [110]. They evaluated four different types of PLLA scaffolds: (a) aligned and (b) randomly oriented microfibers whose diameters were $\sim 1.6 \mu \mathrm{m}$ and (c) aligned and (d) randomly oriented nanofibers whose diameters were $\sim 0.6 \mu \mathrm{m}$. Pro-inflammatory cytokine secretion was more strongly affected by fiber diameter than by fiber alignment. The use of nanofiber scaffolds attenuated the secretion of pro-inflammatory molecules (TNF $\alpha$, RANTES (regulated on activation, normal T expressed and secreted), granulocyte-colony stimulating factor (G-CSF), and MIP-1 $\alpha$ ) when compared to microfibrous scaffolds and flat films. Furthermore, the angiogenic cytokine VEGF was secreted by the macrophages cultured on all the electrospun scaffolds but not those cultured on flat films. In particular, the scaffolds fabricated with randomly oriented nanofibers sustained the secretion of VEGF during 7 days of culture.

Ballota et al. also evaluated electrospun scaffolds for the effect of cyclic strain on the polarization of macrophages towards the M1 or M2 phenotype [109]. They found that the degree of strain $(0,7$, or $12 \%)$ has a significant effect on macrophage phenotype. Macrophages with M1 markers were present at all the tested strain degrees. M2 macrophages were also present in scaffolds subjected to 0 and $7 \%$ strain (Figure 4E), and their numbers increased over time. The gene expression of pro-inflammatory (MCP-1, IL-6, and TNF $\alpha$ ) and anti-inflammatory (IL-10) markers was also evaluated in this study. The treatment at $7 \%$ strain showed the highest levels of expression for all the genes evaluated compared to the unstrained and $12 \%$-strained samples. This study also demonstrates that macrophages subjected to strain forces are capable of the same 
deposition of collagen type III and sulfated glycosaminoglycans as seen in macrophages cultured in unstrained scaffolds.

The importance of physical cues in determining cell fate and behavior has been well established in recent years. However, our understanding of the fine details is incomplete, and more research is needed on this topic. In general, it is not a trivial task to cleanly discriminate the effects of shape, size, roughness, mechanical properties, and chemical composition in a biological experimental setting. Consequently, some of the available experimental evidence on the effect of particular physical cues in macrophage behavior is inconclusive or conflicting. The design of scaffolds for tissue engineering applications, particularly for M1-M2 modulation, will be highly influenced by the notion of the influence of physical cues in the years to come.

\section{Applications, challenges, and the road ahead}

The controlled released strategies discussed here can be applied in diverse tissue engineering and regenerative medicine scenarios and can be further extended to other non-tissue engineering areas, including treatment of autoimmune diseases, metabolic syndrome, etc.

Tissue engineering applications involving M1-M2 immunomodulation of vascularization and bone regeneration are receiving (and will continue to receive) remarkable attention. The roles of the various macrophage populations, particularly M1 and M2, in vascularization and angiogenesis are currently controversial and poorly understood [111]. In general, evidence supports the idea that M1 macrophages initiate vascularization [112], but the role of M2 macrophages is less well understood. M1 macrophage-secreted factors, such as IFN $\gamma$, TNF $\alpha$, IL$1 \beta$, and VEGF, are known to enhance blood vessel sprouting by inducing the proliferation of endothelial cells, whereas signaling from M2 macrophages is known to promote vascular 
anastomosis through secretion of PDGF-BB [113-115]. However, monocytes/macrophages can also inhibit angiogenesis and neovascularization in different diseases and in response to Wntcalcineurin-Flt1 signaling [116-118].

Inflammation, angiogenesis, and bone tissue regeneration are closely tied together, and the proper signaling sequence is critical for normal bone healing [119]. Therefore, scaffolds for vascularized bone have been designed that specifically control macrophage responses by tuning the release of specific cytokines and drugs (Table 1) [28,62,64-66]. Recent studies suggest that inflammatory-related signals from M1 and M2 macrophage phenotypes can enhance bone tissue regeneration by promoting angiogenesis and vascularization [28]. However, further investigation is still needed to determine the actual interplay between M1 and M2 macrophages that regulates angiogenesis during the bone tissue healing process.

Autoimmune diseases are another area in which the use of controlled release immunomodulatory strategies could be of great help in clinical practice. One of the challenges in treating autoimmune diseases is the inability to reach specific cells in target tissues to deliver a specific drug. In this context, nanotechnology strategies can be highly useful. The various drug delivery approaches that have been proposed or assayed for the treatment of several common autoimmune diseases have been recently reviewed by Yuan et al. [120]. For example, recent evidence indicates that type 2 diabetes is an inflammatory disease; thus, immunomodulation has been suggested as a viable strategic treatment approach [121].

The application of immunomodulation based on controlled release strategies to different biological scenarios must be accompanied by more fundamental research. Our understanding of the interplay between implants and the immune response is still incomplete. The use of state-ofthe-art high-throughput gene expression profiling of cell (or tissue) responses to 
immunomodulating biomaterials could reveal a more complete and clearer picture of the interconnection between relevant biochemical signals during inflammation and healing. The use of genetic profiling has recently been initiated in this context [4].

Table 1. Controlled release studies that affect the M1-M2 balance in vascularization and bone regeneration.

\begin{tabular}{|c|c|c|c|c|}
\hline Materials & $\begin{array}{l}\text { Controlled release } \\
\text { methods }\end{array}$ & Cytokine / Drug & Role and in vivo Effect & Reference \\
\hline \multirow{2}{*}{$\begin{array}{l}\text { Decellularized bone } \\
\text { scaffold }\end{array}$} & \multirow{2}{*}{$\begin{array}{l}\text { Biotin-avidin } \\
\text { binding }\end{array}$} & $\mathrm{IFN} \gamma$ & $\begin{array}{c}\text { Promotes M1 macrophages } \\
\text { Increases blood vessels in } \\
\text { vivo }\end{array}$ & \multirow[t]{2}{*}[28]{} \\
\hline & & IL-4 & Promotes M2 macrophages & \\
\hline Collagen scaffold & Adsorption & $\begin{array}{l}\text { Lipopolysaccharide } \\
\text { (LPS) }\end{array}$ & $\begin{array}{l}\text { Promotes M1 macrophages } \\
\text { No vascularization in vivo } \\
\text { but high inflammatory cell } \\
\text { infiltration into scaffolds }\end{array}$ & [62] \\
\hline $\begin{array}{l}\text { poly(L-lactic acid) } \\
\text { oligomer grafted } \\
\text { gelatin hydrogels }\end{array}$ & $\begin{array}{c}\text { Dissolved in } \\
\text { poly(L-lactic acid }) \\
\text { oligomer grafted } \\
\text { gelatin }\end{array}$ & $\begin{array}{c}\text { SEW2871 } \\
\text { (Sphingosine-1 } \\
\text { phosphate (S1P) } \\
\text { receptor agonist) }\end{array}$ & $\begin{array}{l}\text { Promotes macrophage } \\
\text { migration } \\
\text { When combined with } \\
\text { platelet-rich plasma (PRP), } \\
\text { increases bone regeneration } \\
\text { in vivo compared to either } \\
\text { agent alone }\end{array}$ & [66] \\
\hline $\begin{array}{c}\text { Nanofibers of } \\
\text { poly(DL-lactide-co- } \\
\text { glycolide) (PLGA) } \\
\text { and polycaprolactone } \\
\text { (PCL) }\end{array}$ & $\begin{array}{l}\text { Dissolved in } \\
\text { PCL/PLGA }\end{array}$ & $\begin{array}{l}\text { FTY720 } \\
\text { (S1P receptor } \\
\text { agonist) }\end{array}$ & $\begin{array}{c}\text { Promotes M2 macrophages } \\
\text { Increases vascularization } \\
\text { and bone regeneration in } \\
\text { vivo }\end{array}$ & [65] \\
\hline PLGA thin film & $\begin{array}{l}\text { Simple loading } \\
\text { in film }\end{array}$ & $\begin{array}{l}\text { FTY720 } \\
\text { (S1P receptor } \\
\text { agonist) }\end{array}$ & $\begin{array}{c}\text { Promotes M2 macrophages } \\
\text { Increases arteriolar diameter } \\
\text { and capillary tortuosity in } \\
\text { vivo }\end{array}$ & [64] \\
\hline
\end{tabular}

The development of new and smarter materials for M1-M2 modulation is another clear area of development in the immediate future. The need for new materials implies the parallel development (or refinement) of the micro- and nanofabrication technologies now available. The use of microfabrication techniques such as 3D printing, micromolding, and photopatterning will expand the spectrum of possibilities to produce smarter biomaterials for immunomodulation in 
tissue engineering and other applications. And yet, even simpler strategies remain to be explored. For instance, the use of anti-TNF $\alpha$ antibody fragments (instead of the more costly full length anti-TNF $\alpha$ mAbs) could simplify (and significantly lower the cost of) the controlled release platforms now available to control inflammation. Other immuno-modulatory peptides (not necessarily $\mathrm{mAb}$ fragments) promise to add significantly to our toolbox to control inflammation and promote healing. In addition, the use of small molecules in controlled release applications to mitigate inflammation by interfering with or blocking $\mathrm{TNF} \alpha$ has not been reported. However, recent publications demonstrate that small anti-TNF $\alpha$ drugs can effectively mitigate inflammation (by different mechanisms) when administered systemically or applied topically. These molecules might be the next generation of anti-inflammatory drugs and could hold great potential in the context of tissue engineering and regenerative medicine, niches in which their use has not been explored yet [39,122-125].

\section{Acknowledgments}

MMA gratefully acknowledges the institutional funding received from Tecnológico de Monterrey (seed funding to Strategic Research Groups, 2015) and funding provided from CONACyT (Consejo Nacional de Ciencia y Tecnología, México) in the form of Scholarships to GTdS (postdoctoral scholarship 234713) and MMA (sabbatical scholarship 262130). MMA, GTdS and AK acknowledge funding from MIT International Science and Technology Initiatives (MISTI) and the Tecnológico de Monterrey-MIT Program. JCL acknowledges funding from NIH (NIDCR R03DE021755 and NIAMS R21AR065644) and the American Heart Association Scientist Development Grant (12SDG8980014). GTdS acknowledges funding from Fundación México en Harvard. AMG acknowledges funding from the UK Engineering and Physical Sciences Research Council (EP/N006615/1). AK acknowledges funding from the National 
Science Foundation (EFRI-1240443), IMMODGEL (602694), and the National Institutes of Health (EB012597, AR057837, DE021468, HL099073, AI105024, AR063745).

\section{Literature Cited}

[1] S. Franz, S. Rammelt, D. Scharnweber, J.C. Simon, Immune responses to implants - A review of the implications for the design of immunomodulatory biomaterials, Biomaterials. 32 (2011) 6692-709. doi:10.1016/j.biomaterials.2011.05.078.

[2] M.J. Webber, O.F. Khan, S.A. Sydlik, B.C. Tang, R. Langer, A perspective on the clinical translation of scaffolds for tissue engineering, Ann. Biomed. Eng. 43 (2014) 641-56. doi:10.1007/s10439-014-1104-7.

[3] O. Veiseh, J.C. Doloff, M. Ma, A.J. Vegas, H.H. Tam, A.R. Bader, et al., Size- and shapedependent foreign body immune response to materials implanted in rodents and nonhuman primates, Nat. Mater. 14 (2015) 643-51. doi:10.1038/nmat4290.

[4] T. Ehashi, T. Takemura, N. Hanagata, T. Minowa, H. Kobayashi, K. Ishihara, et al., Comprehensive genetic analysis of early host body reactions to the bioactive and bio-inert porous scaffolds, PLoS One. 9 (2014) e85132. doi:10.1371/journal.pone.0085132.

[5] S.F. Badylak, T.W. Gilbert, Immune response to biologic scaffold materials, Semin. Immunol. 20 (2008) 109-16. doi:10.1016/j.smim.2007.11.003.

[6] J.F. Mano, G.A. Silva, H.S. Azevedo, P.B. Malafaya, R.A. Sousa, S.S. Silva, et al., Natural origin biodegradable systems in tissue engineering and regenerative medicine: Present status and some moving trends, J. R. Soc. Interface. 4 (2007) 999-1030. doi:10.1098/rsif.2007.0220.

[7] C.D. Mills, L.L. Lenz, K. Ley, Macrophages at the fork in the road to health or disease, Front. Immunol. 6 (2015) 59. doi:10.3389/fimmu.2015.00059.

[8] L. Chávez-Galán, M.L. Olleros, D. Vesin, I. Garcia, Much More than M1 and M2 Macrophages, There are also CD169(+) and TCR(+) Macrophages., Front. Immunol. 6 (2015) 263. doi:10.3389/fimmu.2015.00263.

[9] J.A. Hamilton, P.P. Tak, The dynamics of macrophage lineage populations in inflammatory and autoimmune diseases, Arthritis Rheum. 60 (2009) 1210-21. doi:10.1002/art.24505.

[10] L.E. Lucca, D.A. Hafler, Sodium-activated macrophages: The salt mine expands, Cell Res. 25 (2015) 885-6. doi:10.1038/cr.2015.91.

[11] P. Robbe, C. Draijer, T.R. Borg, M. Luinge, W. Timens, I.M. Wouters, et al., Distinct macrophage phenotypes in allergic and nonallergic lung inflammation., Am. J. Physiol. Lung Cell. Mol. Physiol. 308 (2015) L358-67. doi:10.1152/ajplung.00341.2014.

[12] K. Pappas, A.I. Papaioannou, K. Kostikas, N. Tzanakis, The role of macrophages in 
obstructive airways disease: Chronic obstructive pulmonary disease and asthma, Cytokine. 64 (2013) 613-25. doi:10.1016/j.cyto.2013.09.010.

[13] A. Mantovani, S. Sozzani, M. Locati, P. Allavena, A. Sica, Macrophage polarization: Tumor-associated macrophages as a paradigm for polarized M2 mononuclear phagocytes, Trends Immunol. 23 (2002) 549-55.

[14] A. Mantovani, A. Sica, Macrophages, innate immunity and cancer: Balance, tolerance, and diversity, Curr. Opin. Immunol. 22 (2010) 231-7. doi:10.1016/j.coi.2010.01.009.

[15] G. Solinas, G. Germano, A. Mantovani, P. Allavena, Tumor-associated macrophages (TAM) as major players of the cancer-related inflammation, J. Leukoc. Biol. 86 (2009) 1065-73. doi:10.1189/jlb.0609385.

[16] J.C. Zarif, J.R. Hernandez, K.F. Sachsenmeier, R.E. Hollingsworth, K.J. Pienta, Targeting M2-tumor associated macrophages (M2-TAMs) in prostate cancer, Cancer Res. 75 (2015) 2365-2365. doi:10.1158/1538-7445.AM2015-2365.

[17] M.Q. Nicol, B.M. Dutia, The role of macrophages in influenza A virus infection, Future Virol. 9 (2014) 847-862. doi:10.2217/fvl.14.65.

[18] J.H. Campbell, A.C. Hearps, G.E. Martin, K.C. Williams, S.M. Crowe, The importance of monocytes and macrophages in HIV pathogenesis, treatment, and cure, AIDS. 28 (2014) 2175-87. doi:10.1097/QAD.0000000000000408.

[19] F.O. Martinez, L. Helming, S. Gordon, Alternative activation of macrophages: An immunologic functional perspective, Annu. Rev. Immunol. 27 (2009) 451-83. doi:10.1146/annurev.immunol.021908.132532.

[20] S. Epelman, K.J. Lavine, A.E. Beaudin, D.K. Sojka, J.A. Carrero, B. Calderon, et al., Embryonic and adult-derived resident cardiac macrophages are maintained through distinct mechanisms at steady state and during inflammation, Immunity. 40 (2014) 91104. doi:10.1016/j.immuni.2013.11.019.

[21] C. Jakubzick, E.L. Gautier, S.L. Gibbings, D.K. Sojka, A. Schlitzer, T.E. Johnson, et al., Minimal differentiation of classical monocytes as they survey steady-state tissues and transport antigen to lymph nodes, Immunity. 39 (2013) 599-610. doi:10.1016/j.immuni.2013.08.007.

[22] S.J. Jenkins, D. Ruckerl, P.C. Cook, L.H. Jones, F.D. Finkelman, N. van Rooijen, et al., Local macrophage proliferation, rather than recruitment from the blood, is a signature of TH2 inflammation., Science. 332 (2011) 1284-8. doi:10.1126/science.1204351.

[23] M.H. Sieweke, J.E. Allen, Beyond stem cells: self-renewal of differentiated macrophages., Science. 342 (2013) 1242974. doi:10.1126/science.1242974.

[24] L.C. Davies, S.J. Jenkins, J.E. Allen, P.R. Taylor, Tissue-resident macrophages., Nat. Immunol. 14 (2013) 986-95. doi:10.1038/ni.2705.

[25] J. Kzhyshkowska, A. Gudima, V. Riabov, C. Dollinger, P. Lavalle, N.E. Vrana, Macrophage responses to implants: Prospects for personalized medicine, J. Leukoc. Biol. (2015). doi:10.1189/jlb.5VMR0415-166R.

[26] Z. Xia, J.T. Triffitt, A review on macrophage responses to biomaterials, Biomed. Mater. 1 (2006) R1-9. doi:10.1088/1748-6041/1/1/R01.

[27] J. Xue, S. V Schmidt, J. Sander, A. Draffehn, W. Krebs, I. Quester, et al., Transcriptome- 
based network analysis reveals a spectrum model of human macrophage activation, Immunity. 40 (2014) 274-88. doi:10.1016/j.immuni.2014.01.006.

[28] K.L. Spiller, S. Nassiri, C.E. Witherel, R.R. Anfang, J. Ng, K.R. Nakazawa, et al., Sequential delivery of immunomodulatory cytokines to facilitate the M1-to-M2 transition of macrophages and enhance vascularization of bone scaffolds, Biomaterials. 37 (2015) 194-207. doi:10.1016/j.biomaterials.2014.10.017.

[29] P.J. Murray, J.E. Allen, S.K. Biswas, E.A. Fisher, D.W. Gilroy, S. Goerdt, et al., Macrophage Activation and Polarization: Nomenclature and Experimental Guidelines, Immunity. 41 (2014) 14-20. doi:10.1016/j.immuni.2014.06.008.

[30] F. De Paoli, B. Staels, G. Chinetti-Gbaguidi, Macrophage phenotypes and their modulation in atherosclerosis, Circ. J. 78 (2014) 1775-81.

[31] F.O. Martinez, S. Gordon, The M1 and M2 paradigm of macrophage activation: Time for reassessment, F1000Prime Rep. 6 (2014) 13. doi:10.12703/P6-13.

[32] F.Y. McWhorter, T. Wang, P. Nguyen, T. Chung, W.F. Liu, Modulation of macrophage phenotype by cell shape, Proc. Natl. Acad. Sci. U. S. A. 110 (2013) 17253-8. doi:10.1073/pnas.1308887110.

[33] M. Bartneck, K.T. Warzecha, F. Tacke, Therapeutic targeting of liver inflammation and fibrosis by nanomedicine, Hepatobiliary Surg. Nutr. 3 (2014) 364-76. doi:10.3978/j.issn.2304-3881.2014.11.02.

[34] R.E. Mirza, M.M. Fang, W.J. Ennis, T.J. Koh, Blocking interleukin-1 $\beta$ induces a healingassociated wound macrophage phenotype and improves healing in type 2 diabetes., Diabetes. 62 (2013) 2579-87. doi:10.2337/db12-1450.

[35] A. Sindrilaru, T. Peters, S. Wieschalka, C. Baican, A. Baican, H. Peter, et al., An unrestrained proinflammatory M1 macrophage population induced by iron impairs wound healing in humans and mice., J. Clin. Invest. 121 (2011) 985-97. doi:10.1172/JCI44490.

[36] A.K. McNally, J.M. Anderson, Interleukin-4 induces foreign body giant cells from human monocytes/macrophages. Differential lymphokine regulation of macrophage fusion leads to morphological variants of multinucleated giant cells., Am. J. Pathol. 147 (1995) 148799.

[37] N. Wang, H. Liang, K. Zen, Molecular mechanisms that influence the macrophage M1M2 polarization balance, Front. Immunol. 5 (2014) 614. doi:10.3389/fimmu.2014.00614.

[38] D.M. Mosser, J.P. Edwards, Exploring the full spectrum of macrophage activation., Nat. Rev. Immunol. 8 (2008) 958-69. doi:10.1038/nri2448.

[39] D. Brenner, H. Blaser, T.W. Mak, Regulation of tumour necrosis factor signalling: live or let die., Nat. Rev. Immunol. 15 (2015) 362-74. doi:10.1038/nri3834.

[40] T. Horiuchi, H. Mitoma, S. Harashima, H. Tsukamoto, T. Shimoda, Transmembrane TNFalpha: Structure, function and interaction with anti-TNF agents, Rheumatology (Oxford). 49 (2010) 1215-28. doi:10.1093/rheumatology/keq031.

[41] A. Mantovani, A. Sica, S. Sozzani, P. Allavena, A. Vecchi, M. Locati, The chemokine system in diverse forms of macrophage activation and polarization., Trends Immunol. 25 (2004) 677-86. doi:10.1016/j.it.2004.09.015.

[42] X.-M. Meng, P.M.-K. Tang, J. Li, H.Y. Lan, Macrophage Phenotype in Kidney Injury and 
Repair, Kidney Dis. (2015). doi:10.1159/000431214.

[43] G.S. Ashcroft, M.-J. Jeong, J.J. Ashworth, M. Hardman, W. Jin, N. Moutsopoulos, et al., Tumor necrosis factor-alpha (TNF- $\alpha)$ is a therapeutic target for impaired cutaneous wound healing., Wound Repair Regen. 20 38-49. doi:10.1111/j.1524-475X.2011.00748.x.

[44] P.C. Taylor, M. Feldmann, Anti-TNF biologic agents: Still the therapy of choice for rheumatoid arthritis, Nat. Rev. Rheumatol. 5 (2009) 578-82. doi:10.1038/nrrheum.2009.181.

[45] P.A. van Schouwenburg, T. Rispens, G.J. Wolbink, Immunogenicity of anti-TNF biologic therapies for rheumatoid arthritis, Nat. Rev. Rheumatol. 9 (2013) 164-72. doi:10.1038/nrrheum.2013.4.

[46] T. Bongartz, A.J. Sutton, M.J. Sweeting, I. Buchan, E.L. Matteson, V. Montori, Anti-TNF antibody therapy in rheumatoid arthritis and the risk of serious infections and malignancies: Systematic review and meta-analysis of rare harmful effects in randomized controlled trials, JAMA. 295 (2006) 2275-85. doi:10.1001/jama.295.19.2275.

[47] D.W. Grainger, Controlled-release and local delivery of therapeutic antibodies, Expert Opin. Biol. Ther. 4 (2004) 1029-44. doi:10.1517/14712598.4.7.1029.

[48] M.F. Shamji, P. Hwang, R.W. Bullock, S.B. Adams, D.L. Nettles, L.A. Setton, Release and activity of anti-TNF $\alpha$ therapeutics from injectable chitosan preparations for local drug delivery, J. Biomed. Mater. Res. B. Appl. Biomater. 90 (2009) 319-26. doi:10.1002/jbm.b.31289.

[49] E.E. Friedrich, L.T. Sun, S. Natesan, D.O. Zamora, R.J. Christy, N.R. Washburn, Effects of hyaluronic acid conjugation on anti-TNF- $\alpha$ inhibition of inflammation in burns, J. Biomed. Mater. Res. A. 102 (2014) 1527-36. doi:10.1002/jbm.a.34829.

[50] K.S. Foong, R. Patel, A. Forbes, R.M. Day, Anti-tumor necrosis factor-alpha-loaded microspheres as a prospective novel treatment for Crohn's disease fistulae, Tissue Eng. Part C. Methods. 16 (2010) 855-64. doi:10.1089/ten.TEC.2009.0599.

[51] S. Marquette, C. Peerboom, A. Yates, L. Denis, I. Langer, K. Amighi, et al., Stability study of full-length antibody (anti-TNF alpha) loaded PLGA microspheres, Int. J. Pharm. 470 (2014) 41-50. doi:10.1016/j.ijpharm.2014.04.063.

[52] B.R. Carrillo-Conde, R.J. Darling, S.J. Seiler, A.E. Ramer-Tait, M.J. Wannemuehler, B. Narasimhan, Sustained release and stabilization of therapeutic antibodies using amphiphilic polyanhydride nanoparticles, Chem. Eng. Sci. 125 (2015) 98-107. doi:10.1016/j.ces.2014.08.015.

[53] Ö. Erdemli, S. Özen, D. Keskin, A. Usanmaz, E.D. Batu, B. Atilla, et al., In vitro evaluation of effects of sustained anti-TNF release from MPEG-PCL-MPEG and PCL microspheres on human rheumatoid arthritis synoviocytes, J. Biomater. Appl. 29 (2014) 524-42. doi:10.1177/0885328214535958.

[54] B. Bartok, G.S. Firestein, Fibroblast-like synoviocytes: Key effector cells in rheumatoid arthritis, Immunol. Rev. 233 (2010) 233-55. doi:10.1111/j.0105-2896.2009.00859.x.

[55] Q. Wang, H. Li, Y. Xiao, S. Li, B. Li, X. Zhao, et al., Locally controlled delivery of TNF $\alpha$ antibody from a novel glucose-sensitive scaffold enhances alveolar bone healing in diabetic conditions, J. Control. Release. 206 (2015) 232-42. 
doi:10.1016/j.jconrel.2015.03.019.

[56] A. Singh, M. Talekar, A. Raikar, M. Amiji, Macrophage-targeted delivery systems for nucleic acid therapy of inflammatory diseases, J. Control. Release. 190 (2014) 515-30. doi:10.1016/j.jconrel.2014.04.021.

[57] V. Carvalho, P. Castanheira, T.Q. Faria, C. Gonçalves, P. Madureira, C. Faro, et al., Biological activity of heterologous murine interleukin-10 and preliminary studies on the use of a dextrin nanogel as a delivery system, Int. J. Pharm. 400 (2010) 234-42. doi:10.1016/j.ijpharm.2010.08.040.

[58] H. Braat, M.P. Peppelenbosch, D.W. Hommes, Interleukin-10-based therapy for inflammatory bowel disease, Expert Opin. Biol. Ther. 3 (2003) 725-31. doi:10.1517/14712598.3.5.725.

[59] V.A. Kumar, N.L. Taylor, S. Shi, N.C. Wickremasinghe, R.N. D’Souza, J.D. Hartgerink, Self-assembling multidomain peptides tailor biological responses through biphasic release, Biomaterials. 52 (2015) 71-8. doi:10.1016/j.biomaterials.2015.01.079.

[60] J. Gómez-Rodríguez, B. Stijlemans, G. De Muylder, H. Korf, L. Brys, M. Berberof, et al., Identification of a parasitic immunomodulatory protein triggering the development of suppressive M1 macrophages during African trypanosomiasis, J. Infect. Dis. 200 (2009) 1849-60. doi:10.1086/648374.

[61] J. Ratanavaraporn, H. Furuya, Y. Tabata, Local suppression of pro-inflammatory cytokines and the effects in BMP-2-induced bone regeneration, Biomaterials. 33 (2012) 304-16. doi:10.1016/j.biomaterials.2011.09.050.

[62] K.L. Spiller, R.R. Anfang, K.J. Spiller, J. Ng, K.R. Nakazawa, J.W. Daulton, et al., The role of macrophage phenotype in vascularization of tissue engineering scaffolds, Biomaterials. 35 (2014) 4477-88. doi:10.1016/j.biomaterials.2014.02.012.

[63] J.D. Roh, R. Sawh-Martinez, M.P. Brennan, S.M. Jay, L. Devine, D.A. Rao, et al., Tissueengineered vascular grafts transform into mature blood vessels via an inflammationmediated process of vascular remodeling, Proc. Natl. Acad. Sci. U. S. A. 107 (2010) 4669-74. doi:10.1073/pnas.0911465107.

[64] A.O. Awojoodu, M.E. Ogle, L.S. Sefcik, D.T. Bowers, K. Martin, K.L. Brayman, et al., Sphingosine 1-phosphate receptor 3 regulates recruitment of anti-inflammatory monocytes to microvessels during implant arteriogenesis, Proc. Natl. Acad. Sci. U. S. A. 110 (2013) 13785-90. doi:10.1073/pnas.1221309110.

[65] A. Das, C.E. Segar, B.B. Hughley, D.T. Bowers, E.A. Botchwey, The promotion of mandibular defect healing by the targeting of S1P receptors and the recruitment of alternatively activated macrophages, Biomaterials. 34 (2013) 9853-62. doi:10.1016/j.biomaterials.2013.08.015.

[66] Y.-H. Kim, H. Furuya, Y. Tabata, Enhancement of bone regeneration by dual release of a macrophage recruitment agent and platelet-rich plasma from gelatin hydrogels, Biomaterials. 35 (2014) 214-24. doi:10.1016/j.biomaterials.2013.09.103.

[67] D. Pohlers, J. Brenmoehl, I. Löffler, C.K. Müller, C. Leipner, S. Schultze-Mosgau, et al., TGF-beta and fibrosis in different organs - molecular pathway imprints., Biochim. Biophys. Acta. 1792 (2009) 746-56. doi:10.1016/j.bbadis.2009.06.004. 
[68] D.P. Vasconcelos, M. Costa, I.F. Amaral, M.A. Barbosa, A.P. Águas, J.N. Barbosa, Development of an immunomodulatory biomaterial: Using resolvin D1 to modulate inflammation, Biomaterials. 53 (2015) 566-73. doi:10.1016/j.biomaterials.2015.02.120.

[69] D.P. Vasconcelos, M. Costa, I.F. Amaral, M.A. Barbosa, A.P. Águas, J.N. Barbosa, Modulation of the inflammatory response to chitosan through M2 macrophage polarization using pro-resolution mediators, Biomaterials. 37 (2015) 116-23. doi:10.1016/j.biomaterials.2014.10.035.

[70] W. He, G.C. McConnell, T.M. Schneider, R. V. Bellamkonda, A novel anti-inflammatory surface for neural electrodes, Adv. Mater. 19 (2007) 3529-3533. doi:10.1002/adma.200700943.

[71] N. Benkirane-Jessel, P. Lavalle, F. Meyer, F. Audouin, B. Frisch, P. Schaaf, et al., Control of monocyte morphology on and response to model surfaces for implants equipped with anti-inflammatory agent, Adv. Mater. 16 (2004) 1507-1511. doi:10.1002/adma.200306613.

[72] P. Schultz, D. Vautier, L. Richert, N. Jessel, Y. Haikel, P. Schaaf, et al., Polyelectrolyte multilayers functionalized by a synthetic analogue of an anti-inflammatory peptide, $\alpha-$ MSH, for coating a tracheal prosthesis, Biomaterials. 26 (2005) 2621-30. doi:10.1016/j.biomaterials.2004.06.049.

[73] D.P. Go, J.A. Palmer, S.L. Gras, A.J. O’Connor, Coating and release of an antiinflammatory hormone from PLGA microspheres for tissue engineering, J. Biomed. Mater. Res. A. 100 (2012) 507-17. doi:10.1002/jbm.a.33299.

[74] D.P. Go, J.A. Palmer, G.M. Mitchell, S.L. Gras, A.J. O’Connor, Porous PLGA microspheres tailored for dual delivery of biomolecules via layer-by-layer assembly, J. Biomed. Mater. Res. A. 103 (2015) 1849-63. doi:10.1002/jbm.a.35319.

[75] N. Kamaly, G. Fredman, M. Subramanian, S. Gadde, A. Pesic, L. Cheung, et al., Development and in vivo efficacy of targeted polymeric inflammation-resolving nanoparticles, Proc. Natl. Acad. Sci. U. S. A. 110 (2013) 6506-11. doi:10.1073/pnas.1303377110.

[76] G. Fredman, N. Kamaly, S. Spolitu, J. Milton, D. Ghorpade, R. Chiasson, et al., Targeted nanoparticles containing the proresolving peptide Ac2-26 protect against advanced atherosclerosis in hypercholesterolemic mice, Sci. Transl. Med. 7 (2015) 275ra20. doi:10.1126/scitranslmed.aaa1065.

[77] G. Leoni, P.-A. Neumann, N. Kamaly, M. Quiros, H. Nishio, H.R. Jones, et al., Annexin A1-containing extracellular vesicles and polymeric nanoparticles promote epithelial wound repair, J. Clin. Invest. 125 (2015) 1215-27. doi:10.1172/JCI76693.

[78] I. Locatelli, S. Sutti, A. Jindal, M. Vacchiano, C. Bozzola, C. Reutelingsperger, et al., Endogenous annexin A1 is a novel protective determinant in nonalcoholic steatohepatitis in mice, Hepatology. 60 (2014) 531-44. doi:10.1002/hep.27141.

[79] D.H.M. Kusters, M.L. Chatrou, B.A.G. Willems, M. De Saint-Hubert, M. Bauwens, E. van der Vorst, et al., Pharmacological treatment with Annexin A1 reduces atherosclerotic plaque burden in LDLR-/- mice on Western type diet, PLoS One. 10 (2015) e0130484. doi:10.1371/journal.pone.0130484.

[80] A.L. Zachman, S.W. Crowder, O. Ortiz, K.J. Zienkiewicz, C.M. Bronikowski, S.S. Yu, et 
al., Pro-angiogenic and anti-inflammatory regulation by functional peptides loaded in polymeric implants for soft tissue regeneration, Tissue Eng. Part A. 19 (2013) 437-47. doi:10.1089/ten.TEA.2012.0158.

[81] M.I. Bury, N.J. Fuller, J.W. Meisner, M.D. Hofer, M.J. Webber, L.W. Chow, et al., The promotion of functional urinary bladder regeneration using anti-inflammatory nanofibers, Biomaterials. 35 (2014) 9311-21. doi:10.1016/j.biomaterials.2014.07.057.

[82] C.-C. Lin, A.T. Metters, K.S. Anseth, Functional PEG-peptide hydrogels to modulate local inflammation induced by the pro-inflammatory cytokine TNFa, Biomaterials. 30 (2009) 4907-14. doi:10.1016/j.biomaterials.2009.05.083.

[83] J. Su, B.-H. Hu, W.L. Lowe, D.B. Kaufman, P.B. Messersmith, Anti-inflammatory peptide-functionalized hydrogels for insulin-secreting cell encapsulation, Biomaterials. 31 (2010) 308-14. doi:10.1016/j.biomaterials.2009.09.045.

[84] S.-M. Ong, S.K. Biswas, S.-C. Wong, MicroRNA-mediated immune modulation as a therapeutic strategy in host-implant integration, Adv. Drug Deliv. Rev. 88 (2015) 92-107. doi:10.1016/j.addr.2015.05.013.

[85] S. Wirtz, M.F. Neurath, Gene transfer approaches for the treatment of inflammatory bowel disease, Gene Ther. 10 (2003) 854-60. doi:10.1038/sj.gt.3302013.

[86] M.D. Bhavsar, M.M. Amiji, Oral IL-10 gene delivery in a microsphere-based formulation for local transfection and therapeutic efficacy in inflammatory bowel disease, Gene Ther. 15 (2008) 1200-9. doi:10.1038/gt.2008.67.

[87] C. Kriegel, M.M. Amiji, Dual TNF- $\alpha /$ Cyclin D1 gene silencing with an oral polymeric microparticle system as a novel strategy for the treatment of inflammatory bowel disease, Clin. Transl. Gastroenterol. 2 (2011) e2. doi:10.1038/ctg.2011.1.

[88] R.M. Boehler, R. Kuo, S. Shin, A.G. Goodman, M.A. Pilecki, R.M. Gower, et al., Lentivirus delivery of IL-10 to promote and sustain macrophage polarization towards an anti-inflammatory phenotype, Biotechnol. Bioeng. 111 (2014) 1210-21. doi:10.1002/bit.25175.

[89] M. Zhang, Y. Gao, K. Caja, B. Zhao, J.A. Kim, Non-viral nanoparticle delivers small interfering RNA to macrophages in vitro and in vivo, PLoS One. 10 (2015) e0118472. doi:10.1371/journal.pone.0118472.

[90] F. Al-Barwani, B. Donaldson, S.J. Pelham, S.L. Young, V.K. Ward, Antigen delivery by virus-like particles for immunotherapeutic vaccination, Ther. Deliv. 5 (2014) 1223-40. doi:10.4155/tde.14.74.

[91] D.S. Wilson, G. Dalmasso, L. Wang, S. V Sitaraman, D. Merlin, N. Murthy, Orally delivered thioketal nanoparticles loaded with TNF- $\alpha$-siRNA target inflammation and inhibit gene expression in the intestines, Nat. Mater. 9 (2010) 923-8. doi:10.1038/nmat2859.

[92] Z. Huang, J. Gan, L. Jia, G. Guo, C. Wang, Y. Zang, et al., An orally administrated nucleotide-delivery vehicle targeting colonic macrophages for the treatment of inflammatory bowel disease, Biomaterials. 48 (2015) 26-36. doi:10.1016/j.biomaterials.2015.01.013.

[93] H. Lim, J. Noh, Y. Kim, H. Kim, J. Kim, G. Khang, et al., Acid-degradable cationic 
poly(ketal amidoamine) for enhanced RNA interference in vitro and in vivo, Biomacromolecules. 14 (2013) 240-7. doi:10.1021/bm301669e.

[94] L.B. Jensen, J. Griger, B. Naeye, A.K. Varkouhi, K. Raemdonck, R. Schiffelers, et al., Comparison of polymeric siRNA nanocarriers in a murine LPS-activated macrophage cell line: Gene silencing, toxicity and off-target gene expression, Pharm. Res. 29 (2012) 66982. doi:10.1007/s11095-011-0589-0.

[95] F.J. Sheedy, Turning 21: Induction of miR-21 as a key switch in the inflammatory response, Front. Immunol. 6 (2015) 19. doi:10.3389/fimmu.2015.00019.

[96] M. Bartneck, V.A. Schulte, N.E. Paul, M. Diez, M.C. Lensen, G. Zwadlo-Klarwasser, Induction of specific macrophage subtypes by defined micro-patterned structures, Acta Biomater. 6 (2010) 3864-72. doi:10.1016/j.actbio.2010.04.025.

[97] J.D. Waterfield, T.A. Ali, F. Nahid, K. Kusano, D.M. Brunette, The effect of surface topography on early NFאB signaling in macrophages, J. Biomed. Mater. Res. A. 95 (2010) 837-47. doi:10.1002/jbm.a.32857.

[98] M. Mohiuddin, H.-A. Pan, Y.-C. Hung, G.S. Huang, Control of growth and inflammatory response of macrophages and foam cells with nanotopography, Nanoscale Res. Lett. 7 (2012) 394. doi:10.1186/1556-276X-7-394.

[99] E.M. Sussman, M.C. Halpin, J. Muster, R.T. Moon, B.D. Ratner, Porous implants modulate healing and induce shifts in local macrophage polarization in the foreign body reaction, Ann. Biomed. Eng. 42 (2014) 1508-16. doi:10.1007/s10439-013-0933-0.

[100] A.K. Blakney, M.D. Swartzlander, S.J. Bryant, The effects of substrate stiffness on the in vitro activation of macrophages and in vivo host response to poly(ethylene glycol)-based hydrogels, J. Biomed. Mater. Res. A. 100 (2012) 1375-86. doi:10.1002/jbm.a.34104.

[101] M. Bartneck, K.-H. Heffels, Y. Pan, M. Bovi, G. Zwadlo-Klarwasser, J. Groll, Inducing healing-like human primary macrophage phenotypes by 3D hydrogel coated nanofibres, Biomaterials. 33 (2012) 4136-46. doi:10.1016/j.biomaterials.2012.02.050.

[102] K. Garg, N.A. Pullen, C.A. Oskeritzian, J.J. Ryan, G.L. Bowlin, Macrophage functional polarization (M1/M2) in response to varying fiber and pore dimensions of electrospun scaffolds, Biomaterials. 34 (2013) 4439-4451. doi:10.1016/j.biomaterials.2013.02.065.

[103] H.M. Rostam, S. Singh, N.E. Vrana, M.R. Alexander, A.M. Ghaemmaghami, Impact of surface chemistry and topography on the function of antigen presenting cells, Biomater. Sci. 3 (2015) 424-41. doi:10.1039/c4bm00375f.

[104] S. Lee, J. Choi, S. Shin, Y.-M. Im, J. Song, S.S. Kang, et al., Analysis on migration and activation of live macrophages on transparent flat and nanostructured titanium, Acta Biomater. 7 (2011) 2337-44. doi:10.1016/j.actbio.2011.01.006.

[105] A.K. Refai, M. Textor, D.M. Brunette, J.D. Waterfield, Effect of titanium surface topography on macrophage activation and secretion of proinflammatory cytokines and chemokines, J. Biomed. Mater. Res. A. 70 (2004) 194-205. doi:10.1002/jbm.a.30075.

[106] M.A. Alfarsi, S.M. Hamlet, S. Ivanovski, Titanium surface hydrophilicity modulates the human macrophage inflammatory cytokine response, J. Biomed. Mater. Res. A. 102 (2014) 60-7. doi:10.1002/jbm.a.34666.

[107] K.A. Barth, J.D. Waterfield, D.M. Brunette, The effect of surface roughness on RAW 
264.7 macrophage phenotype, J. Biomed. Mater. Res. A. 101 (2013) 2679-88. doi:10.1002/jbm.a.34562.

[108] F.Y. McWhorter, C.T. Davis, W.F. Liu, Physical and mechanical regulation of macrophage phenotype and function, Cell. Mol. Life Sci. 72 (2015) 1303-16. doi:10.1007/s00018-014-1796-8.

[109] V. Ballotta, A. Driessen-Mol, C.V.C. Bouten, F.P.T. Baaijens, Strain-dependent modulation of macrophage polarization within scaffolds, Biomaterials. 35 (2014) 491928. doi:10.1016/j.biomaterials.2014.03.002.

[110] E. Saino, M.L. Focarete, C. Gualandi, E. Emanuele, A.I. Cornaglia, M. Imbriani, et al., Effect of electrospun fiber diameter and alignment on macrophage activation and secretion of proinflammatory cytokines and chemokines, Biomacromolecules. 12 (2011) 1900-11. doi:10.1021/bm200248h.

[111] J. Kitajewski, Wnts heal by restraining angiogenesis, Blood. 121 (2013) 2381-2. doi:10.1182/blood-2013-01-479063.

[112] S. Willenborg, T. Lucas, G. van Loo, J.A. Knipper, T. Krieg, I. Haase, et al., CCR2 recruits an inflammatory macrophage subpopulation critical for angiogenesis in tissue repair, Blood. 120 (2012) 613-25. doi:10.1182/blood-2012-01-403386.

[113] C. Troidl, G. Jung, K. Troidl, J. Hoffmann, H. Mollmann, H. Nef, et al., The temporal and spatial distribution of macrophage subpopulations during arteriogenesis, Curr. Vasc. Pharmacol. 11 (2013) 5-12.

[114] R.C.A. Sainson, D.A. Johnston, H.C. Chu, M.T. Holderfield, M.N. Nakatsu, S.P. Crampton, et al., TNF primes endothelial cells for angiogenic sprouting by inducing a tip cell phenotype, Blood. 111 (2008) 4997-5007. doi:10.1182/blood-2007-08-108597.

[115] A. Fantin, J.M. Vieira, G. Gestri, L. Denti, Q. Schwarz, S. Prykhozhij, et al., Tissue macrophages act as cellular chaperones for vascular anastomosis downstream of VEGFmediated endothelial tip cell induction, Blood. 116 (2010) 829-40. doi:10.1182/blood2009-12-257832.

[116] R.S. Apte, J. Richter, J. Herndon, T.A. Ferguson, Macrophages inhibit neovascularization in a murine model of age-related macular degeneration, PLoS Med. 3 (2006) e310. doi:10.1371/journal.pmed.0030310.

[117] M.J. Rutkowski, M.E. Sughrue, A.J. Kane, S.A. Mills, S. Fang, A.T. Parsa, Complement and the central nervous system: Emerging roles in development, protection and regeneration, Immunol. Cell Biol. 88 781-6. doi:10.1038/icb.2010.48.

[118] J.A. Stefater, S. Rao, K. Bezold, A.C. Aplin, R.F. Nicosia, J.W. Pollard, et al., Macrophage Wnt-Calcineurin-Flt1 signaling regulates mouse wound angiogenesis and repair, Blood. 121 (2013) 2574-8. doi:10.1182/blood-2012-06-434621.

[119] P.M. Mountziaris, A.G. Mikos, Modulation of the inflammatory response for enhanced bone tissue regeneration, Tissue Eng. Part B. Rev. 14 (2008) 179-86. doi:10.1089/ten.teb.2008.0038.

[120] F. Yuan, G.M. Thiele, D. Wang, Nanomedicine development for autoimmune diseases, Drug Dev. Res. 72 (2011) 703-716. doi:10.1002/ddr.20479.

[121] M.Y. Donath, S.E. Shoelson, Type 2 diabetes as an inflammatory disease, Nat. Rev. 
Immunol. 11 (2011) 98-107. doi:10.1038/nri2925.

[122] D.H. Maurice, H. Ke, F. Ahmad, Y. Wang, J. Chung, V.C. Manganiello, Advances in targeting cyclic nucleotide phosphodiesterases, Nat. Rev. Drug Discov. 13 (2014) 290314. doi: $10.1038 / \mathrm{nrd} 4228$.

[123] G. Schett, D. Elewaut, I.B. McInnes, J.-M. Dayer, M.F. Neurath, How cytokine networks fuel inflammation: Toward a cytokine-based disease taxonomy., Nat. Med. 19 (2013) 822-4. doi:10.1038/nm.3260.

[124] W. Tang, Y. Lu, Q.-Y. Tian, Y. Zhang, F.-J. Guo, G.-Y. Liu, et al., The growth factor progranulin binds to TNF receptors and is therapeutic against inflammatory arthritis in mice., Science. 332 (2011) 478-84. doi:10.1126/science.1199214.

[125] M.M. He, A.S. Smith, J.D. Oslob, W.M. Flanagan, A.C. Braisted, A. Whitty, et al., Smallmolecule inhibition of TNF-alpha., Science. 310 (2005) 1022-5.

doi:10.1126/science.1116304.

[126] S. Epelman, K.J. Lavine, G.J. Randolph, Origin and functions of tissue macrophages, Immunity. 41 (2014) 21-35. doi:10.1016/j.immuni.2014.06.013.

\section{Figure captions}

Figure 1: Prevalent nomenclature and timing of macrophages. (A) Most adult tissues contain naïve tissue-resident macrophages that originated in the embryonic stage [126]. In addition, circulating monocytes can be recruited to a site of injury where they differentiate into various macrophage phenotypes. According to the most prevalent nomenclature, two macrophage phenotypes have been recognized. The M1 phenotype (classically activated or pro-inflammatory) is activated by IFN $\gamma$ and LPS. The M2 phenotype (alternatively activated or anti-inflammatory) is activated by IL-4 and IL-13. Modified from Mantovani et al., 2004 [41]. (B) Parallel events occur during bioengineered tissue formation. The biomaterial implantation (with or without cells) will trigger an immune response, which leads to recruitment of various immune cells and macrophage infiltration. At this stage, a dominant M1 macrophage phenotype should be expected, and pro-inflammatory stimuli (required during the first stage of an injury) are upregulated. Next, a timely progression toward an anti-inflammatory chemical scenario could be 
beneficial for more rapid and smoother implant-tissue integration and healing; thus the M2 phenotype is more desirable in the second stage. (C) A recent report by Xue et al. reported many more than the two widely recognized (M1 and M2) phenotypes: (a) Nine different macrophage phenotypes were identified in human macrophages based on their transcriptome signatures upon activation by different chemical cues. When macrophages were (b) activated with known M1 or M2 chemical cues or (c) with M1 or M2 related chemical signals, they displayed a biochemical behavior consistent with the M1-M2 polarization model. (d) However, activation with other chemical cues (e.g., free fatty acids, high density lipoprotein (HDL), or molecules associated with chronic inflammation) resulted in seven other distinct macrophage phenotypes (a multi-axis spectrum; $\mathrm{C} 1, \mathrm{C} 3, \mathrm{C} 4, \mathrm{C} 5, \mathrm{C} 7, \mathrm{C} 8, \mathrm{C} 9) . \mathrm{C} 2$ and $\mathrm{C} 6$ are consistent with the expression profile of M2 and M1 phenotypes, respectively. Adapted with permission from Xue et al., 2014 [27].

Figure 2. The progression of stages from the M1 to M2 phenotypes can be controlled at various time points through different strategies. (A) A simplified representation of the signaling network relevant to inflammation and healing after implantation of a scaffold. The main actors in the network are represented: TNF $\alpha$, IL-4, and IL-10. M1-M2 polarization can be controlled or disrupted by the controlled release of different molecules that interfere with proinflammatory cytokines (e.g., release of anti- TNF $\alpha$ monoclonal antibodies (mAbs) or proteins) or amplify anti-inflammatory stimuli (e.g., release of IL-10). Micro- and nanoparticles loaded with different chemical agents (e.g., siRNA, DNA, anti- TNF $\alpha$ mAbs, IL-10 and other cytokines, etc.) have been used as controlled-release agents to modulate the M1-M2 balance. (B) The ultimate aspiration would be to design and fabricate "smart tissue engineering scaffolds" with controlled release potential that would regulate the M1-M2 balance (and therefore the healing, 
tissue repair, and vascularization processes). While the M1 macrophage phenotype is needed immediately after injury, and inflammation is an important and required component of the proper response of the human body to injury, a timely shift towards the M2 phenotype is required to complete the healing/tissue regeneration process. (C) The different controlled release strategies reviewed here that aim to tune the M1-M2 equilibrium.

\section{Figure 3. Examples of controlled release strategies used to modulate the M1-M2 balance in} tissue engineering applications. A) Use of a hydrogel scaffold loaded with immunomodulators. Three-dimensional microCT images of bone regeneration in a rat defect model six weeks after implantation of hydrogels loaded with (a) PBS, (b) SEW281, (c) PRP, and (d) SEW287 and PRP are shown. Reprinted with permission from Kim et al., 2014 [66]. (B) Encapsulation of Nterminal peptide consisting of amino acids 2-26 of Annexin A1 (Ac2-26) in a PLGA-PEG nanoparticle conjugated to a peptide targeting collagen IV. Reprinted with permission from Kamaly et al. (2013) [75]. (C) Decellularized small intestinal submucosa (SIS) was coated with anti-inflammatory peptide amphiphiles (AIF-PAs) derived from uteroglobin protein sequences. Five weeks after implantation in a rat bladder augmentation model, SIS coated with peptides 1 or 2 (SIS/AIF-PA1/2) showed decreased numbers of M1 macrophages (labeled as M1+M2- where CD86+ cells were labeled as M1+) compared to uncoated (SIS) scaffolds or scaffolds coated with a control peptide amphiphile (SIS/AIFC-PA6). After five weeks, SIS/AIF-PA1/2 scaffolds had lower total numbers of M1 and M2 cells (M1+M2+ where CD206+ were labeled as M2+) compared to control scaffolds. Top and bottom row of asterisks represent comparisons to SIS and SIS/AIFC-PA6, respectively, and * represents $p<0.05$, ** represents $p<0.01$, *** represents $p<0.001$, and $* * * *$ represents $p<0.0001$. Reprinted from Bury et al. (2014), with permission from Elsevier [81]. (D) Delivery of AF488-siRNA and MIF expression within 
primary macrophages. (a) Confocal microscopy images of macrophage cultures. Nuclei were stained with DAPI (blue), and nanoparticles with siRNA were dyed green. (b) Western blots showing MIF and $\beta$-actin (used for normalization) protein expression in macrophages. (c) qRTPCR analysis of MIF gene expression in macrophages. PBS and scrambled siRNA served as negative controls. Reprinted with permission from Zhang et al., 2015 [89].

Figure 4. Effect of physical cues found in different scaffolds. (A) Cartoon showing the effect of culturing macrophages in $2 \mathrm{D}$ vs. $3 \mathrm{D}$ substrates of the same chemical composition. Reprinted with permission from Bartneck et al., 2012 [101]. (B) Macrophages seeded in scaffolds with nanotopographies composed of nanodots on the order of 10-200 nm. Micrographs show the effect on cell shape, and histograms show the effect on the cytokine and chemokine gene expression profile. Reprinted with permission from Mohiuddin et al., 2012 [98]. (C) Micropatterned scaffolds promote cell elongation. The induced cell shape derives from expression of differential markers and secretion of several cytokines. Reprinted with permission from McWhorter et al., 2013 [32]. (D) Electrospun sheets containing various polymer concentrations form scaffolds with different porosities and surface area. Macrophages respond differently to each scaffold architecture and show M2-like behavior when seeded on scaffolds produced with high concentrations of polymer. Reprinted with permission from Garg et al., 2013 [102]. (E) Macrophages adhering to electrospun scaffolds and subjected to different strain levels. The applied deformation affected the macrophage phenotype and gene expression profile. Reprinted with permission from Ballota et al., 2014 [109]. 